\title{
A Survey on Handover Management in Mobility Architectures
}

\author{
Stefano Ferretti, Vittorio Ghini, Fabio Panzieri \\ Department of Computer Science and Engineering, University of Bologna \\ Mura Anteo Zamboni 7, 40127 Bologna, Italy
}

\begin{abstract}
This work presents a comprehensive and structured taxonomy of available techniques for managing the handover process in mobility architectures. Representative works from the existing literature have been divided into appropriate categories, based on their ability to support horizontal handovers, vertical handovers and multihoming. We describe approaches designed to work on the current Internet (i.e. IPv4-based networks), as well as those that have been devised for the "future" Internet (e.g. IPv6-based networks and extensions). Quantitative measures and qualitative indicators are also presented and used to evaluate and compare the examined approaches. This critical review provides some valuable guidelines and suggestions for designing and developing mobility architectures, including some practical expedients (e.g. those required in the current Internet environment), aimed to cope with the presence of NAT/firewalls and to provide support to legacy systems and several communication protocols working at the application layer.
\end{abstract}

Keywords: Mobility Management, Handover, Mobile Applications, Multi-Homing, Cross-Layer Protocols.

\section{Introduction}

Over the last few years, several architectural solutions have been proposed to support users that connect to the Internet through a Mobile Node (MN). The main objective is to provide seamless communications, i.e. ensuring that if a MN changes its point of attachment to the Internet, while in movement, no communication interruptions are perceived at the application level, and if such interruptions occur, they do not significantly degrade the Quality of Service (QoS) delivered at the

Email addresses: s.ferretti@unibo.it (Stefano Ferretti), vittorio.ghini@unibo.it (Vittorio Ghini), fabio.panzieri@unibo.it (Fabio Panzieri) 
application level. While throughput remains a major goal of system design, the main concern of mobility architectures is how to best manage situations where a MN changes network. This event is currently referred to as handover (or handoff).

By default, current operating systems installed on smartphones adopt the following strategy for data transmission: one Network Interface Card (NIC) at a time is configured and employed to send data. If a WiFi network is available, the terminal switches to $\mathrm{WiFi}$; otherwise a cellular network is utilized, if the latter is available too. During the handover, communications are interrupted. While the widespread use of current smartphones confirms that in general such a simple approach may be a viable solution, in some cases this strategy has some severe limitations. Just as an example (which is actually a true story), let us consider the case of an employee working in an institution/company composed of several buildings, all covered by a WiFi network (e.g. a researcher in a university campus). Suppose that the researcher is a commuter and, just before leaving for going home, he/she receives an important Voice over IP (VoIP) phone call. Since he needs to leave to take the last train home, he decides to answer the call using his/her mobile phone; today, there are plenty of smartphone apps that offer very efficient VoIP services. At that moment, the device is connected through $\mathrm{WiFi}$, but when he gets out of the building, the WiFi signal is lost and the smartphone automatically switches to $4 \mathrm{G}$ without any handover management at the application level, thus experiencing a first communication interruption. While moving, he passes through other buildings (hence, within their WiFi coverage); as a consequence, the smartphone switches back to WiFi (i.e. a second communication interruption occurs), and then back to $4 \mathrm{G}$ (i.e. yet another communication interruption) and so on. One might suggest that the employee should turn off the WiFi NIC before leaving, thus using the cellular NIC only; yet, a full $3 \mathrm{G} / 4 \mathrm{G}$ coverage may not be available in all the various buildings he goes through. Indeed, when moving, there are cases where one needs to change NIC without breaking the communication at the application layer.

Handovers may actually require a change of the Access Point (AP) (cell or WiFi $\mathrm{AP}$ ) due to the MN movement, thus causing a reconfiguration at the datalink layer of the NIC in use (horizontal handover). However, the MN can also change the network technology, switching from one NIC to another, which causes a reconfiguration at higher network layers (vertical handover). Changing network means that the IP address associated to the MN changes as well; this has repercussions on the application layer, since in the current Internet, the IP address of a node usually plays the twofold role of MN locator and MN identifier. This "change of identity" causes a service interruption that requires more time than a simple network reconfiguration at the operating system level.

Various proposals have been put forward to deal with this problem. Some ap- 
proaches described in these proposals suggest a decoupling of the node identifier from its address (locator), e.g. GLI-Split [1], HIP [2, 3], Hi3 [4], ILNP [5], LISP [6], MILSA [7], NIIA [8, 9], RANGI [10]. These approaches usually comply with future Internet visions, requiring some radical changes in the network architecture. Other approaches address the above mentioned "change of identity" issue by exploiting (and enhancing) protocols of the current Internet stack, e.g. ABPS [11], DCCP [12], SIP-IAPP [13, 14], I-TCP [15], MMUSE [16], MPTCP [17, 18], m-SCTP [19], MSOCKS [20], TCP-migrate [21]. These latter approaches can be classified based on their ability to support the use of a single NIC at a time, or the (possibly concurrent) use of multiple NICs. They may work at various levels in the network stack, or even use a cross-layer strategy employing different functionalities at different levels.

The plethora of available proposals reveals that there are many technical issues concerned with the main problem of mobility management, as well as different technologies that need to be supported, and several alternative ways of solving these issues and using the technologies available. There are solutions that may work in principle, but cannot be deployed in practice because, for example, i) the protocols they use do not comply with the current Internet implementation, ii) they cannot deal with the situation in which a node is behind a Network Address Translation (NAT)/firewall, and iii) they do not take into consideration the fact that some important existing applications do not respect the Internet protocol stack stratification. Thus, there is a significant need to identify and state the main issues making up the whole problem, and to classify the possible approaches for mobility management. This paper illustrates these main issues, as well as experiences and lessons learned from systems and proposals available in the literature, and eventually provides a critical discussion that might help practitioners in devising a holistic solution for mobility management support.

In the rest of the paper, we give some background information on host mobility management services, review the main architectural solutions proposed in the literature, and come up with a classification that arranges solutions according to their design principles. We also discuss aspects that have an impact on their deployment in real scenarios and limit their applicability. It is worth mentioning that, while this paper was being written, new studies have been published on the same topic, e.g. [22]. However, these works mainly focus on the aforementioned future generation Internet and on locator-identifier separation mechanisms. Instead, our approach emphasizes multihoming, mobility, the possibility of easily switching from one NIC to another, the compatibility with the existing Internet and problems strictly concerned with the limitations of the current applications and the architectural solutions employed (e.g., presence of NATs, firewalls, violations of the protocol stratification). Moreover, in this paper we focus on host centric net- 
works, i.e. the traditional host-based conversation model that is exploited in the current Internet. Thus, we do not consider neither information-centric networks [23] nor user-centric networks [24, 25].

The main contributions of this work are the following.

1. We provide a comparative overview of the main architectural solutions for mobility support in wireless networks. All the considered systems are classified based on their main characteristics, the features they offer, and the level of network protocol stack they operate.

2. We provide some valuable guidelines for developing mobility architectures in the current Internet, summarised as follows. Firstly, proxies used in many applications (VoIP, Session Initiation Protocol (SIP)-based applications, and optionally HTTP-based applications [26]) should be upgraded/extended to cope with mobility issues. Secondly, NATs and firewalls have to be handled carefully. Thirdly, multihoming solutions should take into account that many widespread applications and related protocols (e.g. SIP-based ones) do violate the layering principles of the protocol stack. Solutions to this problem require the use of an external proxy and/or the modification of application messages.

3. We describe the main quantitative measures and qualitative indicators for evaluating mobility architecture, and classify all the presented approaches accordingly.

The remainder of this paper is structured as follows. Section 2 provides the background information and the basic definitions related to this topic. Section 3 presents the existing architectural solutions working with single NICs, while Section 4 discusses solutions that exploit multiple NICs. Section 5 gives a qualitative comparison of the host mobility architectures discussed in the paper. Finally, Section 6 provides some concluding remarks and the main guidelines for developing mobility architectures.

\section{Main Definitions and Concepts}

The aim of a host mobility architecture is to ensure that a MN can move seamlessly across different access networks, without any interruptions of the active network services. Before going into the details of such architectures, in this section we need to introduce some concepts and terminology that will be used throughout the whole paper. Then, we will describe how handover is executed when a MN changes its AP within the same Internet Service Provider (ISP). We will also discuss how MNs are identified and localized in a network. We will clarify the notion of proxies and relays, which are useful distributed entities for supporting mobility. After that, we will propose a classification of handover management 
schemes to support mobility. From the discussion provided, it will be clear that dealing with NATs and firewalls (for systems working on the current Internet) is absolutely crucial and that several implications arise from the use of session and application layer protocols that cannot be ignored.

\subsection{Definitions}

\section{Handover}

Handover (or handoff) is the process of transferring a network communication when a mobile terminal changes its connection point to the access network (called "point of attachment").

\section{Seamless Handover}

A seamless handover occurs when the handover is performed with no user perceivable interruptions, hence guaranteeing that the user communication session remains active.

\section{Horizontal Handover}

A horizontal handover takes place between points of attachment supporting the same datalink-layer network technology (Figure 1). A user moving from one UMTS cell to another UMTS cell is an example of a horizontal handover.

It is worth mentioning that there are different types of horizontal handovers. For example, in cellular and WiMAX networks, the horizontal handover can be hard or soft.

\section{Hard Handover}

A hard handover is (a horizontal handover) designed to first break off the initial connection with an AP (base station), before switching to another one. Thus, the $\mathrm{MN}$ communicates with one AP at a time only. Connection with the old AP is broken before the new connection is established. A hard handover is also referred to as "break before make" handover, and it should result in a non-perceptible and short interruption (i.e. even if it is a hard handover, it should be seamless).

\section{Soft Handover}

With a soft handover, the connection between the MN and its AP (base station) is retained until a connection is established with another AP. This mechanism is also known as "make before break". In that way, the MN may be connected with two (or even more) APs at a given moment in time.

\section{Vertical Handover}

Vertical handover occurs between points of attachment supporting different datalink-layer network technologies (Figure 2). The switch from WiFi to a cellular network, and vice versa, are examples of vertical handovers. 


\section{Multihoming}

Today, a MN is equipped with multiple NICs (and corresponding IP addresses). Multihoming refers to the possibility that a MN is connected simultaneously to more than one network (Figure 3).

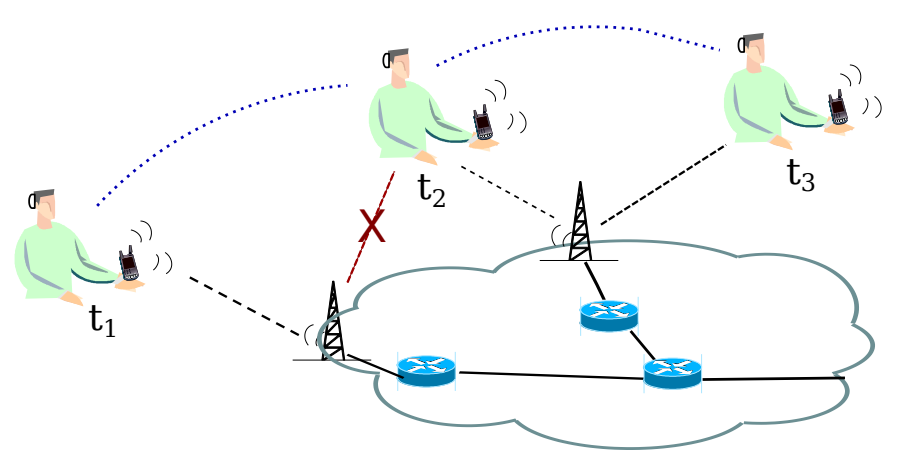

Figure 1: Horizontal Handover: a MN changes access network using the same NIC, while moving

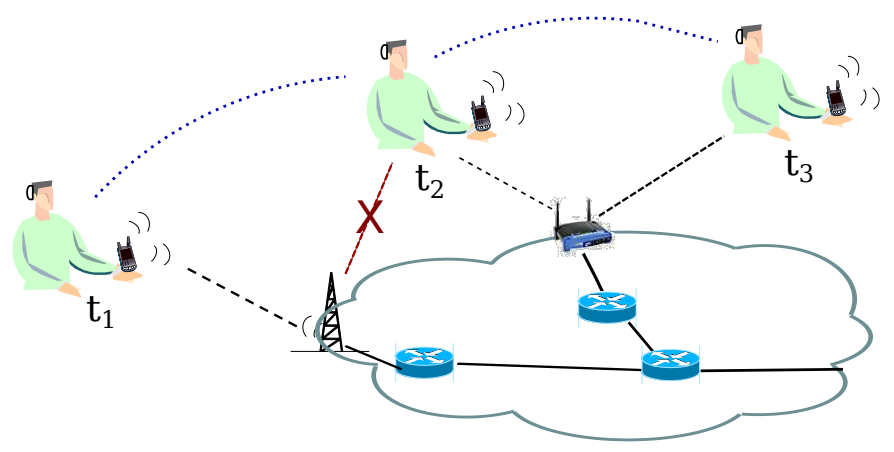

Figure 2: Vertical Handover: a MN changes access network and the NIC used, while moving

Various sophisticated approaches exploit multihoming as a basic solution to increase reliability and offer seamless communications. Ideally, a seamless mobility architecture is responsible for:

1. identifying each given MN univocally;

2. allowing each MN to communicate with its Correspondent Node (CN), (for the purposes of this discussion, a CN might be a fixed or mobile node communicating with the MN);

3. monitoring the QoS provided by different networks, predicting the need of a handover and selecting a new preferred NIC (and the related access point);

4. performing the handover seamlessly, i.e. ensuring the continuity of the communications without any perceivable interruptions for the end users. 


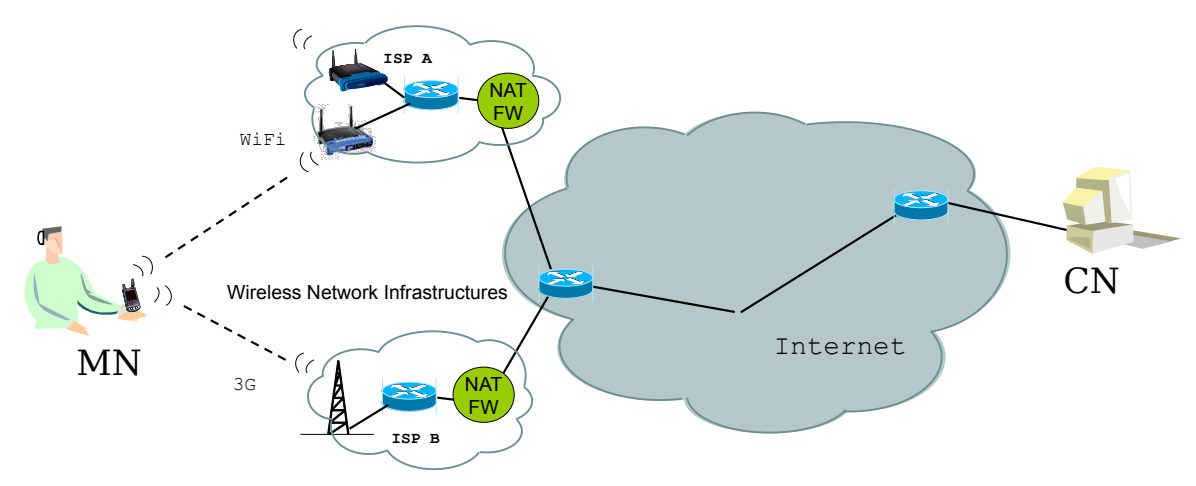

Figure 3: Multihoming: a MN has different active NICs available, ready to be used

\subsection{Intra-ISP Handovers}

This work focuses on systems that deal with the change of network domain, while moving. Thus, we examine mechanisms that allow the connectivity to be maintained when a MN changes its Internet Service Provider (ISP). Before going into the details of such systems, it is important to understand how handovers are handled in current cellular networks and WLANs, when a MN changes its AP within the same ISP. Reconfigurations take place at the physical and data link levels only, while the IP address of the MN remains unchanged.

These mobility management schemes involve the same NIC and the same carrier/network, i.e. they are horizontal handovers. It is worth mentioning, however, that some works refer to the change between $3 \mathrm{G}$ and $4 \mathrm{G}$ as a vertical handover, since, even though the MN employs the same NIC to communicate, there is a change in the type of connectivity used to access a supporting infrastructure [27].

\subsubsection{Cellular Networks}

A cellular network architecture consists of a set of base stations and a core network. The base stations are the APs that provide radio access to MNs, whereas the core network connects them to the wired Internet or the public telephone network. The core network has a very different structure depending on the type of cellular network. For example, $3 \mathrm{G}$ networks such as UMTS support both circuit switched services, used to carry traditional voice communication, and packet switched services for data traffic. The new $4 \mathrm{G}$ Long-Term Evolution (LTE) networks, on the other hand, have been designed to offer packet switched data services only. Voice services should be delivered as data flows over LTE (Voice over LTE - VoLTE). However, due to the high deployment cost and complexity of making such a transition, most $4 \mathrm{G}$ operators currently adopt Circuit-Switched Fallback (CSFB), which switches $4 \mathrm{G}$ users to legacy $3 \mathrm{G}$ and accesses the circuit switched voice service in $3 \mathrm{G}[27]$. 
A detailed description of the cellular communication architecture is out of the scope of this paper. In a few words, a handover performed within the same ISP consists of an interaction between the MN and the core network to select the base station employed to transmit and receive data. In UMTS, for instance, handovers are managed through the Universal Terrestrial Radio Access Network (UTRAN), while LTE uses an Evolved UTRAN (EUTRAN), which simplifies the handover management, thus ensuring lower latencies for handovers and connection setup.

A different procedure is required when the $\mathrm{MN}$ switches between $3 \mathrm{G}$ and $4 \mathrm{G}$. This procedure is divided into two parts, i.e. preparation and execution. During the handover preparation, resources are reserved in the target network. In the execution phase, the $\mathrm{MN}$ is handed over to the target network from the source network. Thus, only the network path needs to be switched, reducing handover processing time. This is done to avoid packet losses, even between radio access systems such as LTE and $3 \mathrm{G}$, which cannot be used simultaneously.

\subsection{2. $W L A N S$}

Here we focus on the horizontal handover performed when a MN switches from an AP of a given WLAN to another AP of the same network, i.e. between APs using the same Service Set Identifier (SSID). The IEEE 802.11 does not specify how to handover from one AP to another one. However, the IEEE released a recommendation called Inter-Access Point Protocol (IAPP) [13], according to which the handover between different APs follows four steps: i) when a MN finds an AP with a signal which is better than the one it is currently attached to, it breaks the connection with the current $\mathrm{AP}$ and sends a reconnection request to the target AP; ii) the target AP establishes a new connection with the MN and sends a notification to the current AP; iii) on reception of this notification, the current AP transfers the MN information to the target AP; iv) finally, the MN switches to the target AP. This is done transparently to the application, and no changes are required at the end points (however, APs must run an implementation of the IAPP protocol). It is worth mentioning that this protocol has been extended to support handovers in different subnets [28] and between different WLANs [14]; the latter solution relies on the use of the SIP protocol.

There are alternative (and in some cases proprietary) solutions to this approach, e.g. the Fast Secure Roaming offered by Cisco, and other scientific proposals such as $[29,30,31]$. Amongst others, it is worth mentioning the IEEE 802.11r-2008, also called Fast Transition, which is an IEEE standard for performing a soft horizontal handover [32]. It is an amendment of the IEEE 802.11 for fast roaming, where the initial handshake with the new AP is done before the MN roams to the target AP. IEEE 802.11r redefines the security key negotiation protocol executed when a MN decides to connect with a new AP. In essence, both the negotiation and the request for wireless resources occur in parallel. This will remove much of the 
handshaking overhead while roaming, thus reducing the handover time between APs while providing security and QoS.

\subsection{Node Localization}

In IP-based communications, the MN's IP address plays the twofold role of MN identifier and locator, as it distinguishes the MN uniquely and identifies its position in the network. When a MN joins a network, it is assigned an IP address that is valid within that network only. When that MN moves to a different network, it acquires a new IP address, thus losing its identity. Hence, it needs to inform CNs of its new identity and location. In general, it is not possible to exclude that a CN will move while communicating with the MN; for that reason, a mobility management architecture must ensure that two hosts can communicate even when they both move and change their addresses simultaneously.

The above problem arises because the IP protocol and its related addressing scheme were originally developed for wired (non-mobile) networks. A number of approaches address this problem by adding and/or modifying services at the network level. In particular, different solutions proposed in the literature attempt to overcome the above problem by separating the node identifier from the node locator $[6,7,33,34,35,36,37]$. The mobile node will therefore have a single identifier that may change over time, regardless of its location.

To summarize, all mobility management architectures adopt some mechanism that:

1. defines a MN identifier uniquely, no matter what the location of the MN is, and

2. provides a localization service that maintains a mapping between the $\mathrm{MN}$ identifier and the current MN location, even when the MN moves.

The localization service is based on a location registry, a service that is assumed to always be available. When a MN changes its IP address, it passes the information to the registry, that is thus informed of the change (registration phase). When a CN wants to initiate a new communication with the $\mathrm{MN}$, or when it wants to continue a communication with the $\mathrm{MN}$ that just changed its address, the $\mathrm{CN}$ starts a lookup phase, asking the location registry for the MN's current address, and then uses the obtained address to contact the MN directly.

This is the general principle employed by different architectures; it may be implemented within different network entities and use very different algorithms and protocols.

\subsection{Presence of Third Distributed Software Entities}

With the aim of managing handovers and the different IP addresses that a MN can obtain while moving, several architectural solutions exploit some distributed 
software components in charge of routing data between the MN and its CN. In the literature, both the term relay and the term proxy are used to refer to these modules. For the sake of precision, we will use both terms, but not as synonyms, in order to emphasize a (maybe subtle but nevertheless important) difference.

\section{Relay}

It is a software entity, which is mainly used to deal with symmetric NATs or firewalls $[38,39,40]$.

Proxy

It is a software entity that is in charge of routing data/information towards a given host, typically in compliance with an application level protocol.

\subsection{Mobility Support and Network Layers}

The architectural solutions we will examine in the rest of this survey are implemented at different levels of the ISO-OSI reference model, as illustrated in Figure 4. This figure illustrates the positioning of the considered systems, in the ISO-OSI reference model, starting from the physical layer (see "PHY" in the bottom part of the figure), datalink layer ("DL"), network layer ("NET"), solutions between the network and transport layers, solutions working at the transport ("TRAN") and session ("SESS") layers. It is worth pointing out that the (brown) circles, placed in the physical and datalink layers in Figure 4, are not mobility management systems; rather, they are criteria/tools exploited to implement mobility management systems. Their description is provided in Section 2.6.

\subsubsection{Architecture Classification}

The architectures reported in Figure 4 can be classified depending on where the localization service is deployed (in Figure 4, a shaded box groups all systems belonging to the same class):

- pure end-to-end solutions distribute the localization service on both the end systems involved in a communication (i.e., MN and $\mathrm{CN}$ );

- home network-based solutions deploy the localization service inside the home network to which the MN belongs;

- access network-based solutions (or border gateway-based solutions) deploy the service inside a border gateway (placed at the edge of each network domain), which the MN exploits as its own access point to the Internet;

- hybrid end-to-end solutions manage the reconfiguration on both the end systems involved in the communication, but place the localization service in a separate server; 


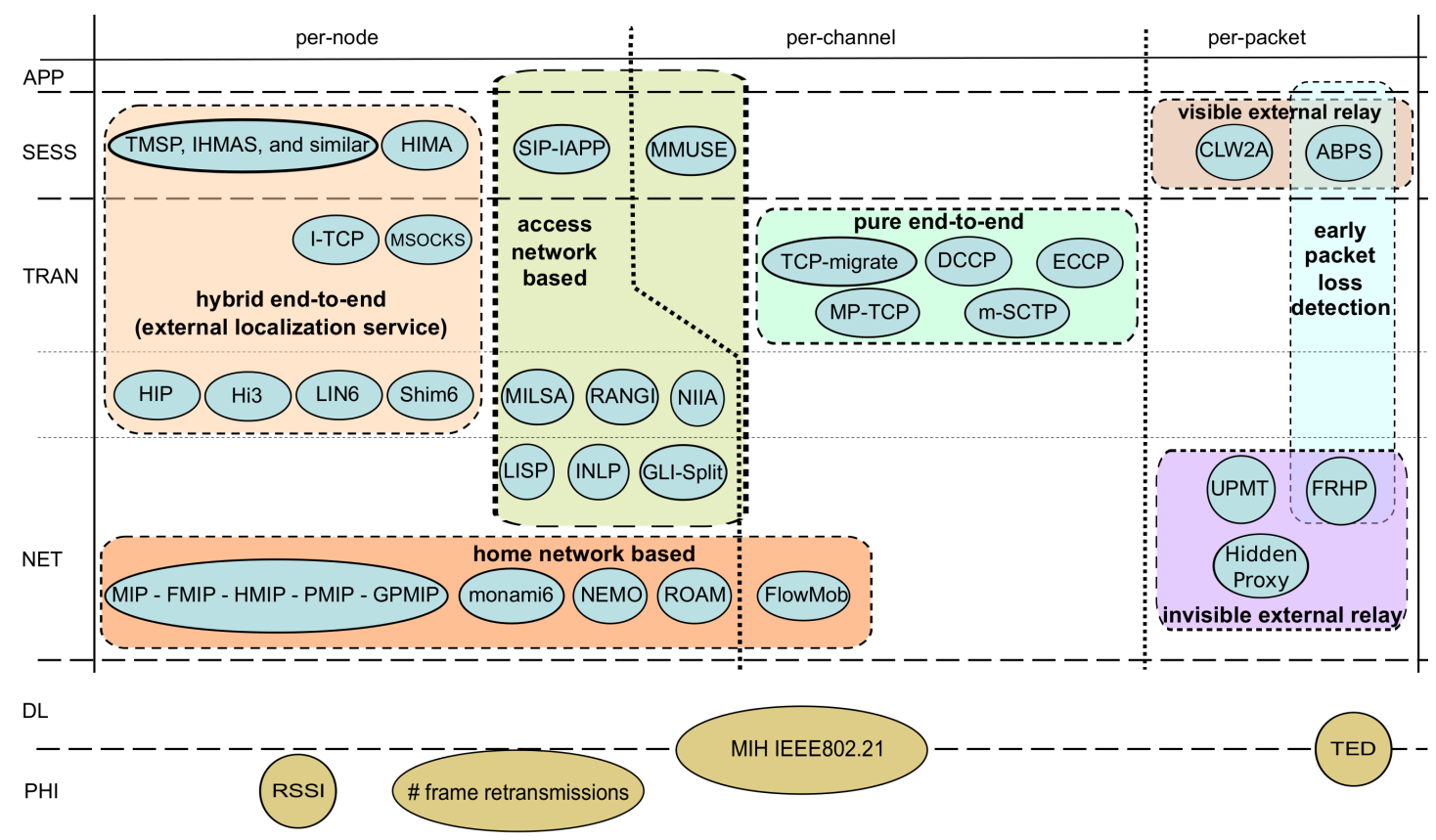

Figure 4: Mobility management architectures

- external relay/proxy solutions integrate both the localization service and the packet relay service at separate servers, which are independent from both the home and access networks. This allows the mobility management service to be deployed with no impact on the network infrastructures, and overcomes the presence of firewalls and NAT systems.

Another important classification relates to the granularity of the service, i.e. to the target (node, channel, packet) to be assigned to a selected NIC. (In Figure 4, systems with the same level of granularity are grouped in the same column.) When triggered, per-node solutions migrate every active flow by adopting a coarsegrained approach and exploiting one NIC only, according to the requirements of the whole node. Conversely, per-channel solutions allow only one flow to be migrated at a time (from one NIC to another) in a finer-grained way compared to the previous approach; this enables each flow to exploit its most suitable NIC. Finally, per-packet solutions route each single IP datagram through the most suitable NIC, allowing fine-grained load-balancing and recovery policies.

In the following sections, we shall examine these solutions individually, according to the ISO-OSI reference layer to which they belong. We will make a distinction between the systems that focus on the use of a single NIC, and those that exploit multihoming and the simultaneous use of multiple NICs to enable MNs to connect to the network. 


\subsection{Tools and Criteria for Mobility Management}

As already mentioned, there are some criteria and tools available at the physical and datalink layers that can be utilized to implement mobility management schemes. This subsection will give a brief outline of each of them.

The Received Signal Strength Indicator (RSSI) is a measurement of the power level being received by the antenna of the NIC. Thus, the higher the RSSI the stronger the signal. Signal strength is one of the main metrics a MN can use in order to discriminate amongst different APs and select the best one to connect to.

Another important criterion is the number of frame retransmissions needed at the lower (Data-Link) layers to deliver a frame from the MN to its AP. Mobility management systems can use this metric as a handover decision criterion.

802.21 is an IEEE standard for dealing with seamless handovers in heterogeneous networks. It has been devised for supporting vertical handovers or, as called in the standard, Media Independent Handovers (MIH). It defines a set of handover-enabling functions to assist MNs during the handovers and handover decision making. Thus, these primitives offered at the operating system level can be exploited to build mobility management architectures. ODTONE is an IEEE 802.21 implementation that is operating-system-independent and open source [41].

Finally, the Transmission Error Detector (TED) is a software tool that provides the MN with information about successful (unsuccessful) datagram reception at the AP [11]. This information can be delivered to software modules working at higher (i.e. application) layers of the protocol stack. In this way, it is possible to devise cross-layer strategies for managing NICs and performing vertical handovers.

\subsection{Coping with NAT and Firewall Systems}

Before going into the details of the systems available in the literature, we shall discuss the possible presence of firewall and NAT systems placed in between endnodes involved in a communication. This is a fundamental issue and one that is often ignored by many mobility management solutions.

Network Address Translators (NATs) are common devices that "hide" private networks behind public IP addresses. A NAT device works by associating a public address and port with a private destination address and port. In essence, the host has a private (and local) transport identifier, which is different from the one that is seen outside the private network. The NAT is responsible for translating the private address into the public address (for outgoing messages) and vice versa (for incoming messages). As a consequence, hosts within a private network behind a NAT are allowed to initiate a communication with a host outside the private network, but quite often the reverse is not possible. Indeed, the CN may not know the public transport identifier (that can be created in real-time by the NAT and change in time). Similarly, many firewalls allow connections to be initiated from 
the private network only, with the effect that these nodes cannot be contacted from other nodes outside the private network to initiate a new communication.

This common practice represents an important limitation for the support of mobility. To cope with this possible situation, the applications can resort to services offered by external Session Traversal Utilities for NATs (STUN) [38], Traversal Using Relays around NAT (TURN) [39] and Interactive Connectivity Establishment (ICE) [40] systems. These are protocols that allow a host (a MN in our case) to learn its public address, as it is seen outside the private network in which it is located. In this way, the MN can activate procedures that enable external hosts to contact it directly, e.g. it can expose its public address in some discovery service.

Unfortunately, the TURN, STUN, ICE protocols do not work correctly when both the end systems ( $\mathrm{MN}$ and $\mathrm{CN}$ ) are protected by (the most common and restrictive) symmetric NATs and firewalls. In this case, each connection of a MN with other nodes is treated separately; moreover, a node does not have a bijective mapping between a local transport-layer address and a public address. This represents a problem, since a typical approach in network programming is to have nodes that employ the same 〈IP address, port t transport address for establishing different transport-level communications (e.g., a node exposes its transport address 〈IP address, port〉 waiting for new connections).

However, the symmetric NAT/firewall maps every communication that has the same internal IP address and port number, locally at the MN, to a different external transport id. Thus, it is not possible to expose a 〈IP address, port $\rangle$ pair to all possible $\mathrm{CNs}$, since only the $\mathrm{CN}$ that receives a packet from a given external transport id representing the MN can send a packet back. The problem is that if each end-system is behind a symmetric NAT, then each system cannot obtain an address to connect to its remote correspondent, as the necessary external transport id of this correspondent has not yet been created by its relative NAT/firewall.

Basically, these restrictive firewalls require an intermediate application-layer relay server, outside any firewall and NAT systems, that receives and forwards the packet exchanged between the $\mathrm{MN}$ and its $\mathrm{CN}$ for the entire duration of the communication (see Figure 5). The relay rewrites the transport and network datagram header so as to appear as the peer node for both MN and CN. In some cases, it is even necessary to rewrite some data inserted into the payload at the application-level. For instance, SIP inserts information on the end point, related to the network and transport levels, within the application payload (e.g., the INVITE message contains the IP address and port of the end-point). Thus, when an end-point is behind a NAT, this information must be modified (we discuss this issue in Section 2.8). The problem is that, as already mentioned, if the $\mathrm{MN}$ is behind a symmetric NAT/firewall, the external IP address and port are assigned only when the application data transmission begins; thus, the INVITE message 
cannot contain such information unless an external transport id is inserted (i.e. the one offered by the relay).

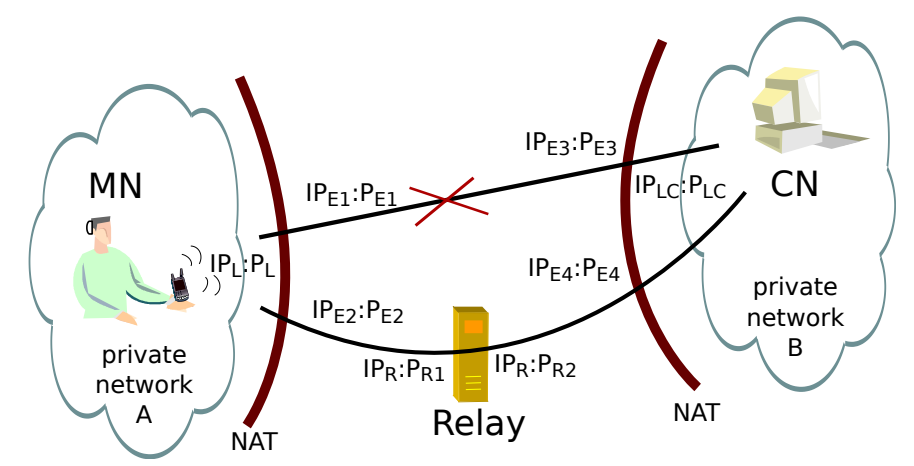

Figure 5: The data relay allows the undirected communication between two nodes behind different firewalls or NAT systems

Figure 6 shows the possible cases that may arise during a communication between a MN and its CN. The main conclusion is that a relay node is needed if we want to guarantee mobility support in any given case. In Figure 6, the proxy represents the software entity which is in charge of managing the MN mobility, provided that the system under consideration uses such a software component. The name "proxy" is generic and it is used in several works, e.g. SIP-based approaches [42, 43, 44, 45, 46], ABPS [11]. As to other approaches, such as MIPv6based approaches (see Section 3.1.1), the proxy in the figure represents the Home Agent (HA).

As shown in Figure 6, a MN and its CN can communicate directly when there is no NAT/firewall constraining the communication (Figure 6(a)). Then, the figure reports the impractical case when the proxy is behind a symmetric NAT/firewall (Figure 6(b)). In this case the MN and the CN cannot contact the proxy; as a result, no communications can be established. Inserting a relay for the proxy might be a solution (Figure 6(c)), but this would certainly be an unusual situation: if we install a proxy in a network, we should be aware that this entity must be reached by hosts outside the private network. Hence, the system designer must take this factor into account.

A more realistic scenario is when the MN connects to the Internet via a private network, which is behind a NAT/firewall (Figure 6(d)). In this case, the MN node can contact the proxy that would act as a relay if the CN asks to connect to the MN. The opposite occurs when the CN is behind a NAT/firewall (Figure 6(e)). In this case, a relay is required to let the MN establish a communication. Indeed, the MN cannot connect to the CN directly, because of the NAT/firewall. The same occurs if a proxy is running for the MN. Moreover, the $\mathrm{CN}$ has to declare that a connection is possible through a relay, at a certain transport address. This is necessary, 
otherwise the $\mathrm{MN}$ is not able to initiate a communication with the CN. However, it is important to point out that in the two last cases, the application must be aware that a relay node has to be contacted; thus, support at the application level is needed to enable the two nodes to interact.

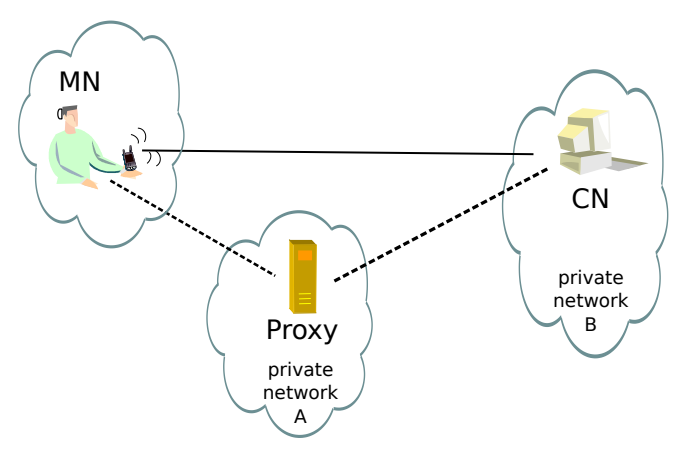

(a) no firewalls

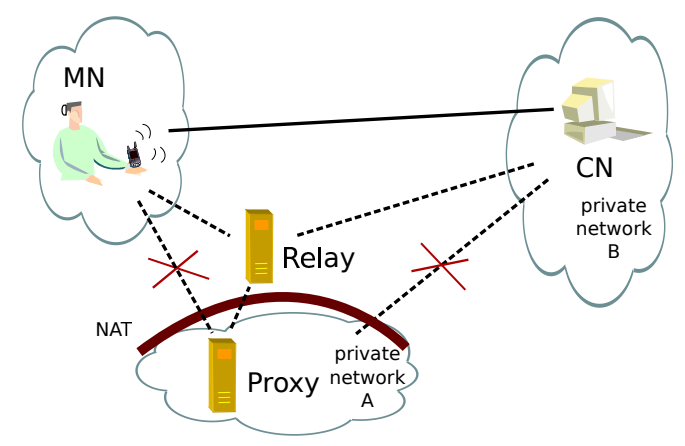

(c) Proxy behind a firewall - use of a relay (impractical situation)

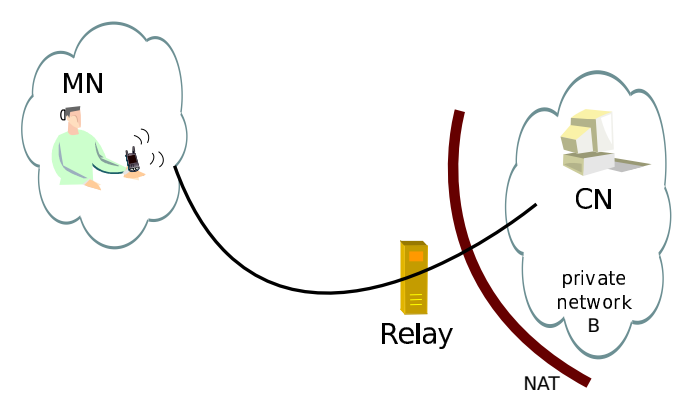

(e) $\mathrm{CN}$ behind a firewall

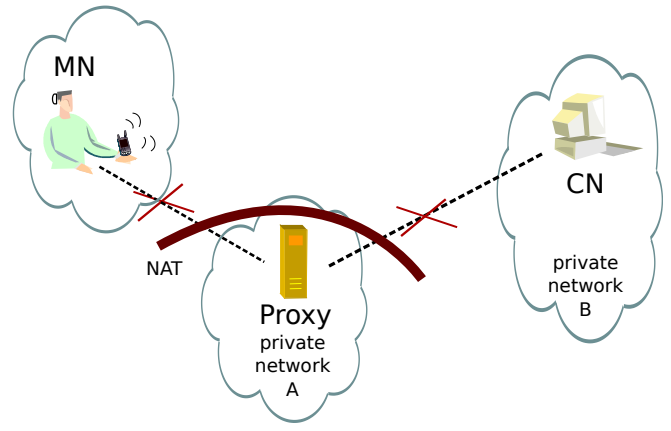

(b) Proxy behind a firewall (impractical situation)

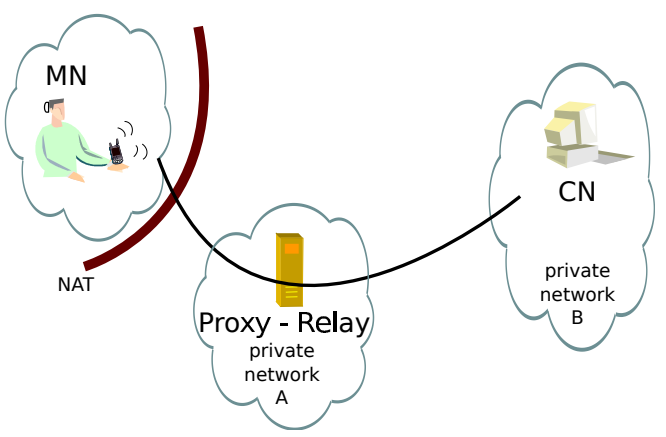

(d) Proxy with relay

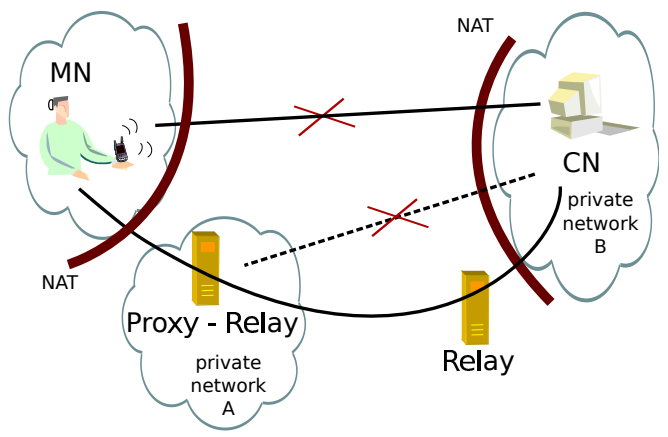

(f) MN and CN behind a firewall

Figure 6: Presence of symmetric NATs/firewalls and need for a relay/proxy. The proxy refers to hosts that enable MNs to communicate while moving (e.g. the Home Agent in MIPv6).

Finally, a further case arises when both $\mathrm{MN}$ and $\mathrm{CN}$ are behind a NAT/fire- 
wall (Figure 6(f)); then, both end-systems must employ a relay to allow their correspondent to contact it.

\subsection{Implications on the Use of Session and Application Layer Protocols}

The considerations reported in the previous subsection suggest that, under certain circumstances, if we want to add mobility and multihoming to current Internet applications, some implementation tricks are needed at the session and application layer protocols. (Hereinafter, we will refer to all protocols working over the transport layer as "application protocols"). There are in fact application-level protocols that

1. do not respect the protocol stack stratification. They typically insert some network and transport layer data into the application payload. There are numerous applications included in this group. For instance, all applications exploiting SIP have these characteristics;

2. respect the separation of roles of protocols in the network stack. Thus, each application message implicitly exploits ports and IP addresses specified in the lower level protocols. HTTP-based applications are examples of this type.

The presence of lower level data in the application payload (first case) represents a problem, firstly, when end-points are behind a NAT or firewall, and secondly, for building a mobility architecture that supports multihoming. Indeed, in the latter situation, there are multiple IP addresses (referring to different NICs) to be managed at the network and at the application layers. The CN might receive different application messages from the MN through multiple IP addresses. Mobility architectures need to cope with this issue.

There are basically two kinds of solutions, which will be presented in the next sections. One refers to the idea of separating the notion of "node identifier" from the "locator" of that node. This type of solution is devised for the so called "Future Internet". Another type of solution requires a proxy. Examples of this second kind are ABPS [11] and all MIPv6-based approaches [47], where the HA (which is a proxy) is responsible for routing messages between the MN and its $\mathrm{CN}$; hence, the CN sees the address of the HA only as its interlocutor. When applications exploit proxies already (e.g. HTTP and SIP-based applications), this solution has a simpler implementation, since the proxy can be upgraded to incorporate the functionalities needed to support multihoming.

\section{Single NIC-based Architectures}

This section focuses on architectural solutions based on the use of a single NIC to manage mobile communications. Table 1 summarizes all the techniques described below, classified according to the protocol layer they operate on. 
Table 1: Single NIC-based Architecture - Protocol Stack Level Classification

\begin{tabular}{|l|c|}
\hline \hline Level & Approaches \\
\hline \hline Session & SIP-IAPP [14] \\
\hline \multirow{2}{*}{ Network } & MIPv6 [47], FMIP [48], HMIP [49], PMIP [50], GPMIP [51], [52], NEMO \\
& [53], LISP [6], ROAM [54] \\
\hline \hline
\end{tabular}

\subsection{Solutions at the Network Layer}

\subsubsection{Mobile IPv6}

Amongst the architectural solutions working at the network layer, it is worth citing the efforts in the Mobile IP version 6 (MIPv6) [47] and its optimizations, e.g. the Fast Handover Mobile IPv6 (FMIP) [48], Hierarchical Mobile IPv6 (HMIP) [49], Proxy Mobile IPv6 (PMIP) [50], GPMIP [51], and [52]. All these approaches employ a Home Agent (HA), i.e. a proxy working inside the access network to which the MN belongs. The HA plays the role of the location registry, and routes datagrams towards the MN when this node is outside its "home network". In addition, if the MN wishes to register its binding with a $\mathrm{CN}$, so as to communicate directly with it, without the interposition of the HA, that MN must perform return routability operations [47]. In order to work properly, MIPv6-based approaches need all the end-systems to have IPv6 capabilities so as to insert some extension headers that transport both the MN's identifier (the home address) and the current MN address into the IP datagrams.

A clear limitation is that these architectural solutions only work on infrastructures with IPv6 capabilities. Moreover, the MIPv6 specification does not allow the simultaneous use of the multiple MN NICs. For each given MN, the address of a single NIC is registered at the HA. In addition, as demonstrated in [55], the handover latency is very high due to the numerous authentication messages, which causes a service disruption time that is not compatible with strongly interactive services such as VoIP.

\subsection{2. $N E M O$}

Network mobility Basic Support Protocol (NEMO BSP or simply NEMO) is concerned with managing the mobility of an entire network. NEMO aims at providing seamless Internet connectivity of the whole mobile network that consists of Mobile Routers (MRs) and MNs. The application scenario is public transportation, such as trains and buses [53]. The network moves around as a whole, along with vehicles. NEMO BSP is based on MIPv6 with minimal extensions. Therefore, the handover mechanism of a MR is essentially the same as that of $\mathrm{MN}$ in MIPv6. In NEMO BSP, a MR serves as a gateway; a permanent address called Home Address (HoA) is obtained on the home link as an identifier of the MR. When the MR moves away, it acquires a Care of Address (CoA) from the access router in the foreign network. MR sends a "binding update" message to its HA 
proxy located in the home network, binding the CoA with the HoA. After the binding process, a bi-directional tunnel is established between the MR and the HA proxy. Packets from the $\mathrm{CN}$ with the destination of MR's HoA are directly routed to the HA, and the HA is in charge of rerouting all packets to the CoA of the MR through a tunnel. MNs in the mobile network have permanent addresses taken from the mobile network prefix advertised on the MR's ingress interface, and packets intended to or originated from the MNs are encapsulated in the tunnel.

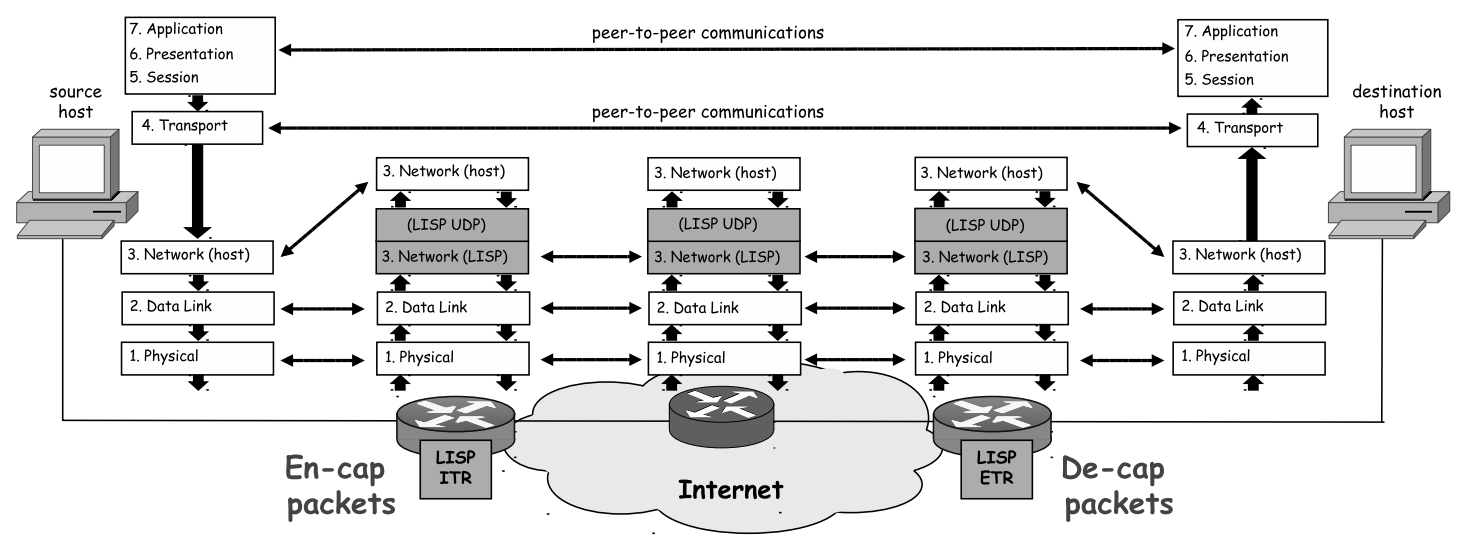

Figure 7: The LISP architecture [6]

\subsection{3. $L I S P$}

Another noteworthy architecture that belongs to the border-gateway class is the Location/ID Separation Protocol (LISP) [6] proposed by Cisco. This is a first solution that produces the aforementioned separation between the identifier of a node, and its current location in the network. As depicted in Figure 7, LISP makes use of an overlay network of LISP routers, located at the edge of the network domains and classified as Ingress Tunnel Router (ITR) and Egress Tunnel Router (ETR). The ITR intercepts the IP datagrams coming from the MNs, maps the sender address (the location) to the sender ID, encapsulates these datagrams in LISP packets and routes them through the LISP routers towards the destination. When a LISP packet reaches the ETR at the edge of the destination domain, the ETR extracts the IP datagram from the LISP packet, maps the destination ID to the destination locator and routes the IP datagram to the destination. In that way, LISP does not require changes to the end systems.

The main drawback of LISP is shared with all other architectures that introduce functionalities at the edge of the network domains, i.e. they need all the domains to be LISP enabled by deploying Ingress/Egress tunnel routers at their 
edges. Indeed, this architecture needs all the paths between each MN and the Internet to flow through at least a LISP-enabled border router. If a MN's NIC connects to a network domain that has no LISP-enabled router at its edge, the LISP architecture fails to provide network continuity to the MN. Recent proposals have been published that provide functionalities for NAT traversal for LISP MNs $[56,57]$.

\subsection{4. $R O A M$}

Some proposals at this level exploit the service provided by Internet Indirection Infrastructure (i3) [58]. i3 builds a rendezvous-based communication abstraction. The system extends point-to-point communications by decoupling the act of sending and the act of receiving messages. The main aim is to provide an approach to ease the development of multicast communications.

In i3, a logical identifier is associated to each node. This identifier can be mapped to the current IP address by means of an indirection point. In this way, when we want to send a message to a $\mathrm{CN}$, the message passes through an i3 server to locate the destination current address.

ROAM [54] exploits and extends i3 to support node mobility. In substance, the indirection point is exploited to manage handovers. A proxy-based solution is adopted to transparently support unmodified applications on mobile nodes. Moreover, the use of an i3 server guarantees that communication is established also in the event of simultaneous handovers of the two mobile nodes.

Support for legacy applications is guaranteed by the introduction of a proxy. However, any application host is required to have a related proxy. Hence, each server should deploy an i3 proxy in order to communicate with the MN. This represents an obvious limitation for the deployability of the system in the current Internet.

Finally, it is worth pointing out that this solution needs a non-negligible amount of additional information to be inserted into packets. Thus, header compression is needed to reduce the packet header overhead.

\subsection{Solutions at the Session Layer}

When solutions are devised to let nodes communicate through different networks, a key role might be played by session protocols that control the dialogue between end-points and incorporate functionalities used by the localization service. Today, Session Initiation Protocol (SIP) is the main protocol employed for controlling multimedia, multi-homed communication sessions [42, 45]. We now review its main characteristics. 


\subsubsection{Session Initiation Protocol (SIP)}

SIP is a session-layer text protocol that uses a message/response handshake for signaling purposes. In particular, it is used to establish or change communication parameters such as IP addresses, protocol ports and audio/video codecs between the end-systems. The SIP specification is extensible and allows application-defined fields to be added to the SIP messages.

The SIP messages worthy of mention here are REGISTER, INVITE and reINVITE. The REGISTER message allows a given node to declare that it is available for communications; it is usually sent to a SIP server that works as the localization service. The INVITE message is used to establish a communication session between two nodes. Typically, this message is sent from the user to the SIP server that replies with the address of the other end node, together with some communication parameters. Then, the two end nodes can communicate directly. A re-INVITE message may be used when communication parameters (such as the IP address) change.

Another important aspect is that the SIP protocol allows the presence of SIP proxies, that can be transparent to the application (proxy agent) or can masquerade the end-systems (Back-to-Back (B2B) user agent) working as an opaque relay.

\subsubsection{SIP and IAPP-based proposals}

A SIP-based mechanism that focuses on WiFi technologies has been proposed that extends the already mentioned IAPP [14]. This approach (hereinafter called SIP-IAPP) proposes a cross-layer approach that modifies the IAPP to speed up the handover in WLANs. This allows direct forwarding of messages addressed to the MN, from an old AP to the new one, while the MN is still reconfiguring its SIP network setting.

This enhancement speeds up the SIP registration, increasing the speed of the handover process as well. However, these approaches again require modifications in the infrastructure, and focus on a single networking technology.

\section{Architectures that Enable Multihoming}

This section reports solutions that cope with handovers by resorting to the possible use of multiple NICs. These schemes work at different levels of the network stack. Table 2 summarizes all the techniques described in this section, classified based on the protocol layer they operate on.

\subsection{Solutions at the Network Layer}

\subsubsection{Monami6}

An extension of MIPv6, called Multiple Care of Address registration (monami6) $[62,63,69,70,71]$ has been proposed for supporting host mobility and 
Table 2: Multihoming Architecture - Protocol Stack Level Classification

\begin{tabular}{|l|c|}
\hline \hline Level & Approaches \\
\hline \hline Cross-Layer & ABPS [11], CLW2A [26] \\
\hline Session & IHMAS [43], TMSP [59], [46], [44], MMUSE [16] \\
\hline \multirow{2}{*}{ Transport } & DCCP [12], m-SCTP [19], TCP-migrate [21], MPTCP \\
& {$[17,18]$, MSOCKS [20], I-TCP [15], ECCP [60] } \\
\hline \multirow{2}{*}{ Between Network and Transport } & HIP [2, 3], Hi3 [4], LIN6 [61], MILSA [7], NIIA [8, 9], \\
& RANGI [10], Shim6 [36] \\
\hline \multirow{2}{*}{ Network } & monami6 [62, 63], FlowMob [64], GSE [65], ILNP [5], \\
& GLI-Split [1], hidden proxy [66], UPMT [67], FRHP [68] \\
\hline
\end{tabular}

multihoming. If a MN configures several IPv6 global addresses on one or more of its NICs, it can register these addresses with its HA as CoAs. This enables the HA to forward messages to the MN.

As for many other solutions, this extension does not overcome firewall and NAT systems, since according to IPv6 based approaches, with the advent of IPv6 there will be no need to employ NAT technologies anymore. In addition, the return routability operations cannot be easily extended to verify multiple CoAs and, as usual, if the CN is protected by a firewall (or NAT system), the return routability operation fails [72].

\subsubsection{FlowMob}

The Flow Mobility technique (FlowMob), described in [64], uses a multiple CoA to enable the MN to register its multiple IP addresses with its HA. This extension allows the MN to separate its outgoing traffic into different flows based on the protocols, port numbers and IP addresses, and forward each given flow using a selected NIC. This technique allows a flow-based switching through the MN's NICs. In [73], the problem of assigning traffic flows to available interfaces is optimized using a heuristic algorithm.

This approach shares the same limitations as the other MIPv6-based approaches: it needs the network infrastructures to be modified in order to add IPv6 capabilities. Moreover, the handover latency is very high, due to the large number of authentication messages. Finally, as mentioned for other IPv6 based strategies, this approach does not provide solutions for symmetric firewalls, and thus requires an external relay to be used.

\subsubsection{Separation between locator and identifier; ILNP}

A completely different scheme refers to the already mentioned idea of modifying the Internet architecture to perform the mapping between the identifier and the locator of a node. One of the first proposals in this direction was the Global, Site, and End-system address elements (GSE) [65]. A prominent example of approaches devised to support mobile nodes is the Identifier Locator Network Protocol (ILNP), where the DNS and ICMP protocols are modified/updated in order to support the 
possible changes of the locator for a given identifier [5]. The approach requires a novel implementation of the network and transport layers at the nodes, while it is compatible with the IPv6 backbone.

Basically, when a MN is moving between two distinct networks, it updates its locator record in the DNS. Thus, if new sessions are established, they are established directly to the node's current location. As regards active sessions, the approach enables the MN to inform its $\mathrm{CN}$ directly of the changes, using newly defined ICMP Locator update messages.

This approach enables multihoming. Moreover, the presence of NATs does not represent a problem, provided that applications employ identifiers only, and no locators, which means that they employ some Application Programming Interfaces (APIs) that do not exploit lower level information (to be implemented in accordance with the new protocols) [74].

Although simple and elegant, these solutions have been devised to work in future Internet scenarios. Thus, this type of approach requires modifications of the infrastructure and its protocols. Moreover, updates of locators at the DNS are not instantaneous; in addition, given the amount of mobile nodes that are on the Internet nowadays, this solution presents some scalability problems (that can be easily solved, but which do have some cost). Hence, this approach may cause some service unavailability, when message updates are lost and both end-points are mobile.

\subsubsection{GLI-Split}

The "Global Locator - Local Locator - Identifier Split" (GLI-Split) is a system that adds a global mapping service to the IPv6 architecture. Locators are distinguished between local ones, employed for local routing within an edge network, and global ones [1]. The mapping system tracks changes for the locators, thus enabling mobility and multihoming. The architecture employs border routers at the edge networks that are in charge of modifying the address information (from local to global and vice versa) contained in packets traversing different edge networks. The system has been designed to work with the existing IPv6 architectures. This solution shares the same drawback mentioned for ILNP, i.e. when the two endpoints move simultaneously and direct updates get lost, a service unavailability can occur.

\subsection{Solutions Between the Network and Transport Layers}

Approaches have been presented in literature that insert an intermediate layer between the network and transport layers of the protocol stack. This layer works on all the end-nodes involved in the communication, i.e., MN and CN (in a one-to-one communication). Notable examples in this class of solutions are the Host Identity Protocol (HIP) [2, 3], Hi3 [4], Location Independent Addressing for IPv6 (LIN6) 
[61, 75], MILSA, [7] and Level 3 Multihoming Shim Protocol for IPv6 (Shim6) [36]. Based on these solutions, the location registry is a DNS-like mapping function that operates as a service outside the access networks and associates host identifiers to host locations.

A limitation of these approaches is the requirement to modify the protocol stack in all the end-nodes involved in a communication. While it is reasonable to require that a $\mathrm{MN}$ installs additional software to support its mobility, the $\mathrm{CN}$ can be a fixed node that may not be interested in supporting the mobility of the MN.

\subsubsection{HIP, Hi3}

In the Host Identity Protocol (HIP), IP addresses are locators used for packets forwarding only, while the concept of host identity is employed as the identifier of a node $[2,3]$. The protocol introduces an "UPDATE" message that can be sent by a MN making a handover to its CN. As mentioned for other approaches, this mechanism fails when both end points are mobile and perform a handover simultaneously. To overcome this limitation, a rendezvous server is exploited that can be queried to map identifiers to their related locators.

The Host Identity Indirection Infrastructure (Hi3) is a networking architecture for mobile nodes, derived from the $\mathrm{i} 3$ and the Host Identity Protocol (HIP) [4, 76]. The basic idea is to allow an IP-based communication while using an indirection infrastructure to route the HIP control messages. The advantages of using i3 as a control plane for HIP in Hi3 include protection from Denial of Service attacks, support for simultaneous mobility, and providing an initial rendezvous service. However, in order for Hi3 to be effective, i3 proxies are required in the network.

\subsection{2. $R A N G I$}

Similarly to HIP, the Routing Architecture for the Next Generation Internet (RANGI) separates location and identifier, adopting an ID/Locator mapping system [10]. In RANGI host locators are ordinary IPv6 addresses and consist of two parts: the first part represents the locator domain, such as the organization affiliation, while the second part is the local host identifier, which is represented as an $\mathrm{IPv} 4$ address (included within the IPv6 address). The mapping system is implemented as a distributed hash table. A node can have multiple locators at the same time with the same identifier (i.e. the IPv4 address) thus enabling multihoming. In this last case, border routers are responsible for modifying the locators, thus enabling multipath delivery strategies. As to mobility, no detailed solutions have been proposed. Similarly to HIP, it is suggested that some update messages can be exploited to inform the $\mathrm{CN}$ of handovers or rehoming. 


\subsubsection{Shim6}

In Shim6, locators are IPv6 addresses, while an upper layer identifier (which is described also as an IPv6 address) is employed to identify end points [36]. As in other approaches, a layer is added in between the network and transport layers. Communication between two end points is performed through a 4-way handshake that allows nodes to determine the locators and upper-level identifiers. To enable mobility, update messages are exploited to change the locators, as in HIP. In case of outage, Shim6 performs an end-to-end exploration of the available addresses using the Reachability Protocol (REAP) [77], and then updates the locators. This approach is not suitable for highly dynamic environments as it is related to timer expiration and not to movement detection.

\subsubsection{MILSA}

MILSA employs the sublayer (added on top of the network layer) to map identifiers exploited at the application layer with network-level locators [7]. Mobility and multihoming might be supported, since the mapper makes changes to the MN's locator which are transparent to the applications. However, some kind of global manager is required for node identifiers. Moreover, no algorithm for the management of handovers is specified.

\subsubsection{NIIA}

Node Identity Inter-networking Architecture (NIIA) employs a node identity layer between the network and transport layers that is exploited to perform routing $[8,9]$. The architecture defines two basic components: locator domains and node identity routers. A locator domain is the abstraction of some kind of local network, having a consistent internal addressing and routing system. Node identity routers perform routing between nodes in different domains. Each node registers to one node identity router. Then, node identity routers are in charge of modifying the headers entering/leaving the local domain, changing the locators so that inter-domain routing is made possible. Moreover, they act as proxies, hence enabling node mobility and multihoming. When a node changes its local domain, a registration phase is executed, and the node identity router of the original local domain of the $\mathrm{MN}$ is informed. Hereinafter, that router will act as a proxy for the MN. In the designed architecture, local domains can be organized in a hierarchical manner. Thus, inter-domain messages can pass through a sequence of local domains; this can introduce additional latencies.

\subsection{Solutions at the Transport Layer}

As for previous approaches, the use of solutions working at the transport-layer may require modifications of the applications on both the $\mathrm{MN}$ and $\mathrm{CN}$ to invoke the services offered by these solutions. An overview of the existing proposals follows. 


\subsection{1. $M N$ as proactive location registry}

The common approach of the protocols working at the transport-layer, such as the datagram-oriented Datagram Congestion Control Protocol (DCCP) [12], the stream-oriented Mobile Stream Control Transport Protocol (m-SCTP) [19], the TCP enhancements TCP-migrate [21] and MultiPath TCP (MPTCP) [17, 18], is as follows. Each given end-system plays the role of a proactive location registry that directly informs the $\mathrm{CN}$ whenever its configuration changes. Unfortunately, this approach may fail when both the end-systems are mobile. Indeed, if they change their IP configuration simultaneously, leaving their current network access point, they would become mutually unreachable. The mentioned schemes support multihoming. While DCCP was originally designed without support for multihoming, some improvements have been proposed to add this feature [78].

\subsection{2. $M S O C K S, I-T C P$}

MSOCKS uses an external proxy that performs TCP connection redirection [20]. Such an external proxy is employed to split the end-to-end communication into two communications: namely MN-proxy and proxy-CN. This solution is referred to as TCP Splice. Hence, MSOCKS uses TCP Splice to migrate a connection when the MN changes its IP address (and potentially when it changes network interface). Since the connection between its $\mathrm{CN}$ and the proxy remains unchanged, the connection will not be interrupted and the $\mathrm{CN}$ will not be aware of the mobility. TCP packets are modified by the external proxy to create the illusion of a single, direct TCP connection between the end-points.

Another example of this kind of approach is I-TCP, that splits the communication into two paths, using an external relay to route messages [15].

\subsection{3. $E C C P$}

The End-to-end Connection Control Protocol (ECCP) is an end-to-end approach that modifies the transport protocol into two sublayers, i.e. i) a connection control, which manages connections, their constituent flows, and their associated addresses, and ii) a data delivery functionality that is responsible for the typical management of the transport flow once a connection is established, like congestion control, flow control, reliability [60]. Thus, functionalities are introduced so that once a node wants to change its NIC, it informs the other node. During the communication, end-points can add flows to an existing connection in order to spread traffic over multiple NICs.

We mentioned that no end-to-end signaling protocol can, by itself, handle the case when both hosts reconfigure or change simultaneously the NIC in use. To sort out this problem, the authors propose the use of a lightweight redirection cache in the local network of either communicating hosts. This cache should keep short-lived redirection state pointing to the new locations of hosts that have 
recently migrated out of its network. When a MN moves, it sends a message to the redirection box of its old network to add a pointer to its new location. This additional scheme requires the modification of the network infrastructure and violates the end-to-end philosophy of the protocol.

\subsection{Solutions at the Session Layer}

\subsubsection{SIP-based approaches}

Several proposals exist that employ SIP to control the session of a multi-homed communication. For instance, the Terminal Mobility Support Protocol (TMSP) [59] exploits an auxiliary SIP server, as location registry placed outside the access networks, that maps a user identifier (e.g. vittorio.ghini@unibo.it) to the current user location (i.e. the IP address of his/her MN). Each MN has a SIP user agent that sends REGISTER messages to the SIP server in order to update its current location. INVITE messages are sent to establish communications with other nodes.

Similarly, [46] presents an architecture capable of managing vertical handoffs, by using a SIP-based approach. The scheme complies to the IP Multimedia Subsystem (IMS), a standardized overlay architecture for session control, authentication, authorization and accountability in all-IP networks. Another related proposal is the one presented in [44] that only supports vertical handoffs from $3 \mathrm{G}$ networks to a WiFi network.

Session-layer solutions might not be efficient as they invoke an external localization service when an IP address reconfiguration occurs. In particular, the SIP-based services introduce an additional delay due to their message/response behavior; in case of reconfiguration, the MN interrupts the communication, sends a SIP signaling message to the $\mathrm{CN}$ and waits for the response before resuming the transmission.

With this in view, the IHMAS work presented in [43] minimizes handoff delays by exploiting a SIP-based, IMS compliant, proactive mechanism that performs registration and renegotiation phases for new connections, while keeping the media flows active over old connections, if these are available.

\subsubsection{Coping with NAT and Firewall Systems}

An example of a system that takes into account the possible presence of firewall and NAT systems is MMUSE [16]. This system requires an auxiliary SIP server (namely, the Session Border Controller, SBC) to be located at the edge of the autonomous system where the MN is entering. This autonomous system may be composed of several subnets using heterogeneous network technologies. While the MN moves across the subnets, each subnet provides the MN with a different IP address. The SBC combines the functionalities of SIP and RTP proxies, firewall and NAT systems, and intercepts the communications that enter and leave the network, in particular the SIP messages between the MN and its CN outside the 
network edge. The SBC sets up the firewall rules that allow the subsequent SIP, RTP and RTCP communications between MN and CN based on the outgoing SIP messages. Moreover, when the MN moves to a different subnet and changes its IP address, the SBC modifies the outgoing datagram, in order to hide the current location of the MN from the CN.

The main limitation of MMUSE is that the MN traffic always needs to flow through a given SBC that resides in the edge of the network. This implies that the MN may only move inside a given autonomous system, but it cannot move across different networks administered by different organizations.

\subsection{Zero impact on Existing Network Infrastructures}

To limit the need for modifying the network infrastructure, the external relay/proxy solutions deploy the localization service at an external proxy, independent from both home and access networks, with no impact on the network infrastructures. This external proxy also incorporates the functionality of data relay to overcome NATs/firewalls. This class of solutions requires some modifications to the MN. Moreover, it splits the communications in two consecutive paths, from the MN to the proxy and from the proxy to the CN (as in all other solutions employing an additional relay to overcome the presence of firewalls).

The external relay/proxy class of host mobility solutions may be divided into two sub-classes: the visible class supports only those applications and protocols (such as Web and VoIP) that define the concept of proxy. It operates using a pair of explicit proxy software components, the one placed inside the MN and the another on the relay/proxy external server. The application at the MN only needs to be configured to use the proxy running on the local host.

On the other hand, the invisible class groups those solutions that operate as a tunnel between the MN and the external proxy; thus, applications running in the $\mathrm{MN}$ are not aware of these software entities. This sub-class does not depend on the application, but handles IP datagrams in different ways, based on the transport protocols. Similarly to the visible class, local proxy (at the MN) and external proxy software components are used. However, in this case, the application in the $\mathrm{MN}$ is unaware of the two proxies; in fact, the $\mathrm{CN}$ recognizes the external proxy server as its correspondent node (i.e., the $\mathrm{MN}$ ).

\subsubsection{Visible Relay/Proxy Services and Early Packet Loss Detection}

These approaches exploit some additional software components on the network that act as proxy and/or relay. Since proxies need to manage application level data, these solutions are application-dependent. Thus, each system supports some specific application or, stated differently, each application protocol requires a specific implementation of the proxy that manages and routes application data. 
The highest-developed system within the visible relay class is the Always Best Packet Switching (ABPS-SIP/RTP) architecture, designed to support applications based on SIP/RTP such as VoIP and Video on Demand (VoD) [11] (Figure 8). In contrast with the previously presented approaches, ABPS-SIP/RTP (or simply, ABPS) enables the transmission of each datagram through the most appropriate NIC among those which are active at the MN. The ABPS-SIP/RTP architecture operates at the session-layer using a so called "proxy client" on the MN and an auxiliary "proxy server" in an external server, allowing the MN to move across different autonomous systems. (The proxy acts as a relay as well, thus overcoming problems due to the presence of NATs and firewalls.) A cross-layer technique is employed to monitor all the concurrent NICs which are available, their performances and those that become active (or inactive); based on their current status, an automatic reconfiguration is performed at the MN. This is accomplished by exploiting an implementation of the previously mentioned TED software module (see Section 2.6).

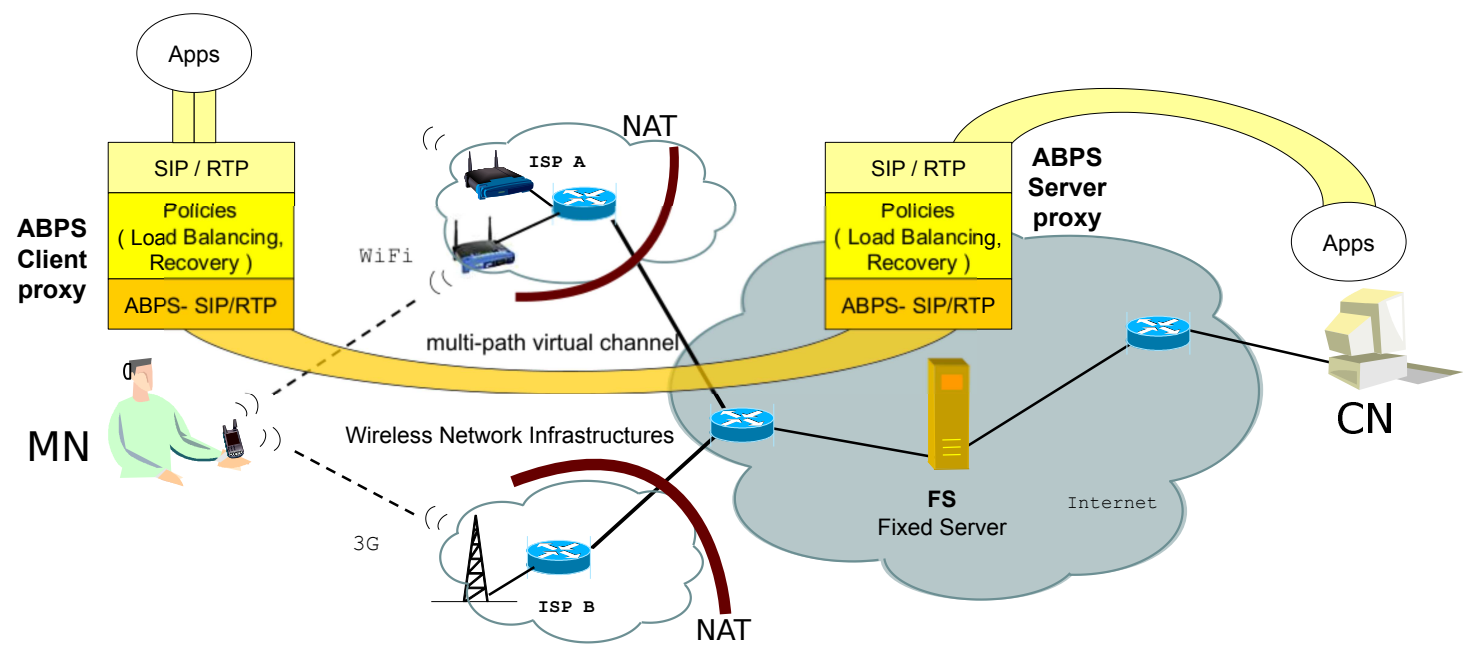

Figure 8: The ABPS-SIP/RTP architecture

This solution uses SIP-compliant proxy servers which interact with additional software modules, installed on the proxies working between the network and the transport layer; these modules are responsible for managing the application-layer data flows, enabling their transmission through different NICs. In practice, an ABPS proxy decides on a per-packet basis which is the best NIC to use to transmit the data. Moreover, each proxy adds a digital signature in the packet so that the proxy server may identify the sender, in spite of its possible different IP addresses. The use of such a signature technique transparently avoids the typical delays introduced by the two way message/response handshake of the SIP signaling phase. 
It is important to note that all the approaches we introduced earlier usually consider the problem of changing a NIC in use as soon as it becomes unavailable. Thus, the decision to change communication technology is not taken based on some particular QoS metric; rather, it is taken based on the failure of the currently adopted NIC. In contrast, ABPS takes into consideration QoS metrics to identify the best NIC to use at any given moment. In particular, a cross-layer mechanism provides the applications with information about the successful (or unsuccessful) datagram transmissions through a given wireless access point.

In essence, the advantages provided by the ABPS-SIP/RTP system are the following:

- it works perfectly over all IP based networks and does not require any modification of the current network infrastructures;

- it is SIP compliant;

- it avoids delays occurring in classic SIP-based approaches when the MN changes its preferred NIC or its configuration. Indeed, in this case other schemes employ reconfiguration phases based on the exchange of INVITE messages, while the ABPS-SIP/RTP approach avoids this additional message exchange;

- it supports RTP-based applications such as VoIP and VoD services;

- it can cope with vertical handoffs, without introducing any additional delay during the passage from the use of a NIC to the next one since, as soon as this becomes available, it is promptly configured to work;

- it optimizes the use of NICs by deciding on a per-packet basis which is the best interface to be used, based on the monitored performances of the available networks and on the employed QoS metrics;

- it overcomes the presence of NATs and firewalls.

The principal limitation of this solution is that the ABPS-SIP/RTP architecture is strictly dedicated to SIP/RTP-based applications and cannot be exploited for other applications.

Another approach that exploits an external proxy-based distributed approach to support HTTP-based Web 2.0 services has been presented in [26]. In Figure 4 it is referred as CLW2A (Cross Layer architecture for Web 2.0 Applications).

These approaches are considered as working on a per-packet basis. Indeed, they work at a level of granularity which is finer than channel flows. However, they do not decide which NIC to use for each packet, since they schedule the NIC 
to use information that is available on top of the transport layer. But they can easily switch from one NIC to another without wasting time in network and system reconfigurations.

\subsubsection{Invisible Relay/Proxy Services}

The visible relay/proxy service solutions are not suitable for applications that are not designed to work with proxies, or more generally for legacy applications. To overcome this limitation, the invisible relay/proxy service class operates transparently from the application standpoint by intercepting messages sent by the applications in the MN and redirecting them to a local proxy. The local proxy delivers each message to the external relay/proxy using all the available NICs of the MN. In the MN, a virtual Ethernet interface is used (i.e. an Ethernet interface not linked to a physical one), and a particular routing table configuration causes the applications to bind their outgoing connections to that virtual interface.

The earliest representative of this class was the "hidden proxy" [66], developed on Linux systems and based on the iptables/netfilter mechanism and on the tun/tap virtual interface. This proxy was dedicated to TCP-based applications [66]. More recently, a similar architecture, called Universal Per-application Mobility management using Tunnels (UPMT), extends to the above mentioned hidden proxy to provide support for UDP-based applications [67]. Both these architectures consider the problem of changing a NIC in use as soon as it becomes unavailable.

Actually, it is conceivable that some solution can be devised that combines the use of an invisible external proxy with the features of early packet loss detection (in Figure 4 and in the following discussion we refer to such a kind of approach as Fast Reactive Hidden Proxy - FRHP). While at the time of writing, there are no available implementations of this strategy to support mobility management, this approach has been utilized in other contexts, such as vehicular networks [68].

\section{Comparison among Host Mobility Architectures}

Tables 3-4 summarize the discussion presented in this paper by providing a concise comparison of the different host mobility architectures we have presented. In both tables, each column refers to one system (or more systems with similar properties). Systems are organized based on the classes identified in the previous sections. Thus, we have pure end-to-end systems, hybrid end-to-end systems, systems employing some software entity placed within the MN's home network, systems that modify the access network, those employing some invisible external relay and, finally, those employing a visible external relay.

This qualitative comparison takes into account different criteria: Table 3 focuses on deployability, systems requirements, and issues concerned with the need 
to modify the applications, nodes or the network. Table 4 focuses on performance issues. Rows display the criteria or features that each solution may have. At a first glance, it can be observed that systems of the same class have similar properties. At the time of writing, there are no available real implementations for some approaches, whereas for others, the related papers/documentation do not provide enough details and do not allow us to retrieve complete information on some performance parameters. Systems for which we do not have enough information are not ranked according to those specific performance metrics, and are instead marked with an "U" (i.e., it is unclear how the system would work in certain conditions).

Tables 3-4 are described in detail as follows. Table 3 is structured in three principal subtables named "General", "Requirements" and "Need Modifications". Table 4 is structured in a single subtable labeled "Performance". These subtables are described below individually. 
Table 3: Comparison among host mobility architectures: Requirements and general issues on the deployability

\begin{tabular}{|c|c|c|c|c|c|c|c|c|c|c|c|c|c|c|c|c|c|c|c|c|c|c|c|c|c|}
\hline & Classes & \multicolumn{2}{|c|}{$\begin{array}{l}\text { Pure } \\
\text { E2E }\end{array}$} & \multicolumn{6}{|c|}{$\begin{array}{c}\text { Hybrid } \\
\text { E2E } \\
\end{array}$} & \multicolumn{3}{|c|}{$\begin{array}{l}\text { Home } \\
\text { Network }\end{array}$} & \multicolumn{9}{|c|}{$\begin{array}{l}\text { Access } \\
\text { Network } \\
\end{array}$} & \multicolumn{3}{|c|}{$\begin{array}{c}\text { Invisible } \\
\text { Proxy }\end{array}$} & \multirow[b]{2}{*}{ 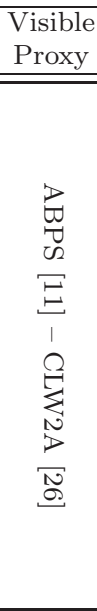 } \\
\hline & Criteria & 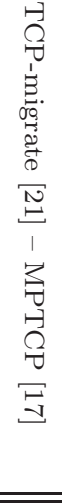 & 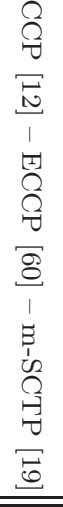 & 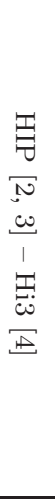 & 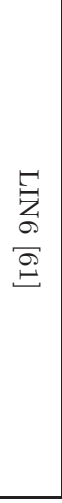 & 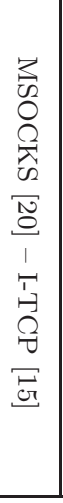 & 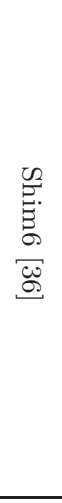 & 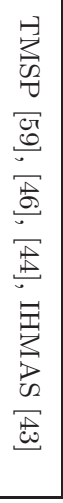 & 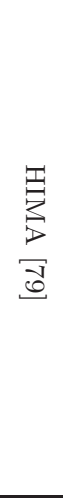 & 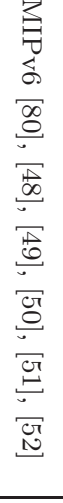 & 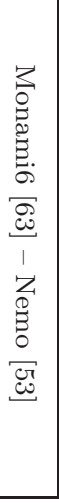 & 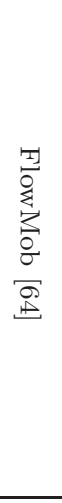 & 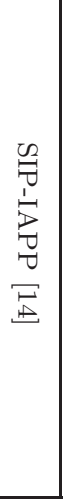 & $\begin{array}{l}\vec{y} \\
\vec{\theta} \\
\sigma\end{array}$ & 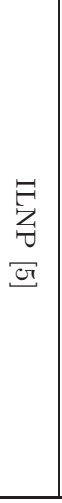 & 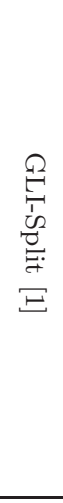 & 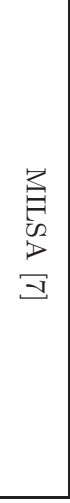 & 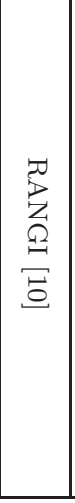 & $\begin{array}{l}\text { 尝 } \\
\infty \\
\infty \\
\bullet\end{array}$ & 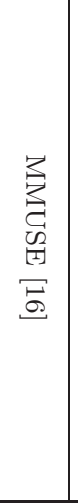 & 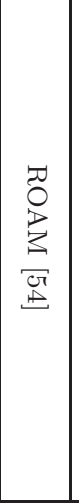 & 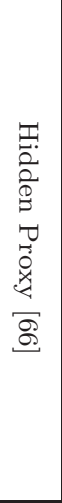 & 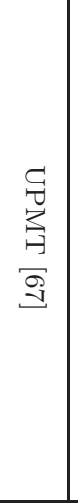 & 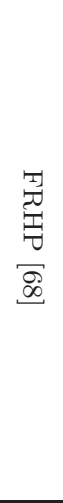 & \\
\hline \multirow{2}{*}{ 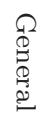 } & Deployability & $\mathrm{Y}$ & $\mathrm{Y}$ & $\mathrm{Y}$ & & $\mathrm{Y}$ & & Y & $\mathrm{Y}$ & & & & Y & & & & & & & Y & $\mathrm{Y}$ & $\mathrm{Y}$ & Y & $\mathrm{Y}$ & Y \\
\hline & Implementation & $\mathrm{Y}$ & Y & $\mathrm{Y}$ & $\mathrm{Y}$ & Y & Y & Y & & $\mathrm{Y}$ & $\mathrm{Y}$ & $\mathrm{Y}$ & Y & $\mathrm{Y}$ & Y & Y & & & Y & Y & & $\mathrm{Y}$ & Y & & $\mathrm{Y}$ \\
\hline \multirow{3}{*}{ 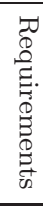 } & $\begin{array}{l}\text { Requires } \\
\text { IPv6 }\end{array}$ & & & & $\mathrm{Y}$ & & $\mathrm{Y}$ & & & $\mathrm{Y}$ & $\mathrm{Y}$ & $\mathrm{Y}$ & & $\mathrm{Y}$ & $\mathrm{Y}$ & $\mathrm{Y}$ & Y & $\mathrm{Y}$ & $\mathrm{Y}$ & & & & & & \\
\hline & $\begin{array}{c}\text { Requires } \\
\text { MIPv66 }\end{array}$ & & & & & & & & & Y & $\mathrm{Y}$ & $\mathrm{Y}$ & & & & & & & & & & & & & \\
\hline & $\begin{array}{c}\text { Requires } \\
\text { IPSec }\end{array}$ & & & & & & & & & $\mathrm{Y}$ & $\mathrm{Y}$ & $\mathrm{Y}$ & & & & & $\mathrm{Y}$ & & & & & $\mathrm{Y}$ & & & \\
\hline \multirow{7}{*}{ 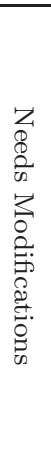 } & $\begin{array}{l}\text { Protocol Stack } \\
\text { in MN }\end{array}$ & $\mathrm{Y}$ & Y & $\mathrm{Y}$ & $\mathrm{Y}$ & Y & $\mathrm{Y}$ & $\mathrm{Y}$ & $\mathrm{Y}$ & Y & $\mathrm{Y}$ & Y & & $\mathrm{Y}$ & $\mathrm{Y}$ & $\mathrm{Y}$ & Y & Y & Y & $\mathrm{Y}$ & Y & $\mathrm{Y}$ & $\mathrm{Y}$ & Y & Y \\
\hline & $\begin{array}{c}\text { Protocol Stack } \\
\text { in CN } \\
\end{array}$ & $\mathrm{Y}$ & $\mathrm{Y}$ & $\mathrm{Y}$ & $\mathrm{Y}$ & $\mathrm{Y}$ & $\mathrm{Y}$ & $\mathrm{Y}$ & $\mathrm{Y}$ & $\mathrm{Y}$ & $\mathrm{Y}$ & $\mathrm{Y}$ & & & $\mathrm{Y}$ & $\mathrm{Y}$ & Y & $\mathrm{Y}$ & $\mathrm{Y}$ & & $\mathrm{Y}$ & & & & \\
\hline & $\begin{array}{c}\text { Applications } \\
\text { in MN } \\
\end{array}$ & & $\mathrm{Y}$ & & & & & $\mathrm{Y}$ & Y & & & & & & $\mathrm{Y}$ & Y & & Y & $\mathrm{Y}$ & & & & & & \\
\hline & $\begin{array}{l}\text { Applications } \\
\text { in CN } \\
\end{array}$ & & $\mathrm{Y}$ & & & & & Y & $\mathrm{Y}$ & & & & & & $\mathrm{Y}$ & $\mathrm{Y}$ & & $\mathrm{Y}$ & Y & & & & & & \\
\hline & $\begin{array}{c}\text { Access } \\
\text { Networks } \\
\end{array}$ & & & & & & & & & $\mathrm{Y}$ & $\mathrm{Y}$ & & $\mathrm{Y}$ & & $\mathrm{Y}$ & $\mathrm{Y}$ & Y & Y & $\mathrm{Y}$ & & & & & & \\
\hline & $\begin{array}{c}\text { Border } \\
\text { Gateways } \\
\end{array}$ & & & & & & & & & & & & Y & Y & Y & Y & Y & Y & Y & Y & & & & & \\
\hline & $\begin{array}{c}\text { Requires } \\
\text { External Relay/Proxy }\end{array}$ & & & & & $\mathrm{Y}$ & & & & & & & & & & & & & & & Y & $\mathrm{Y}$ & $\mathrm{Y}$ & Y & $\mathrm{Y}$ \\
\hline
\end{tabular}


Table 4: Comparison among host mobility architectures: Performance

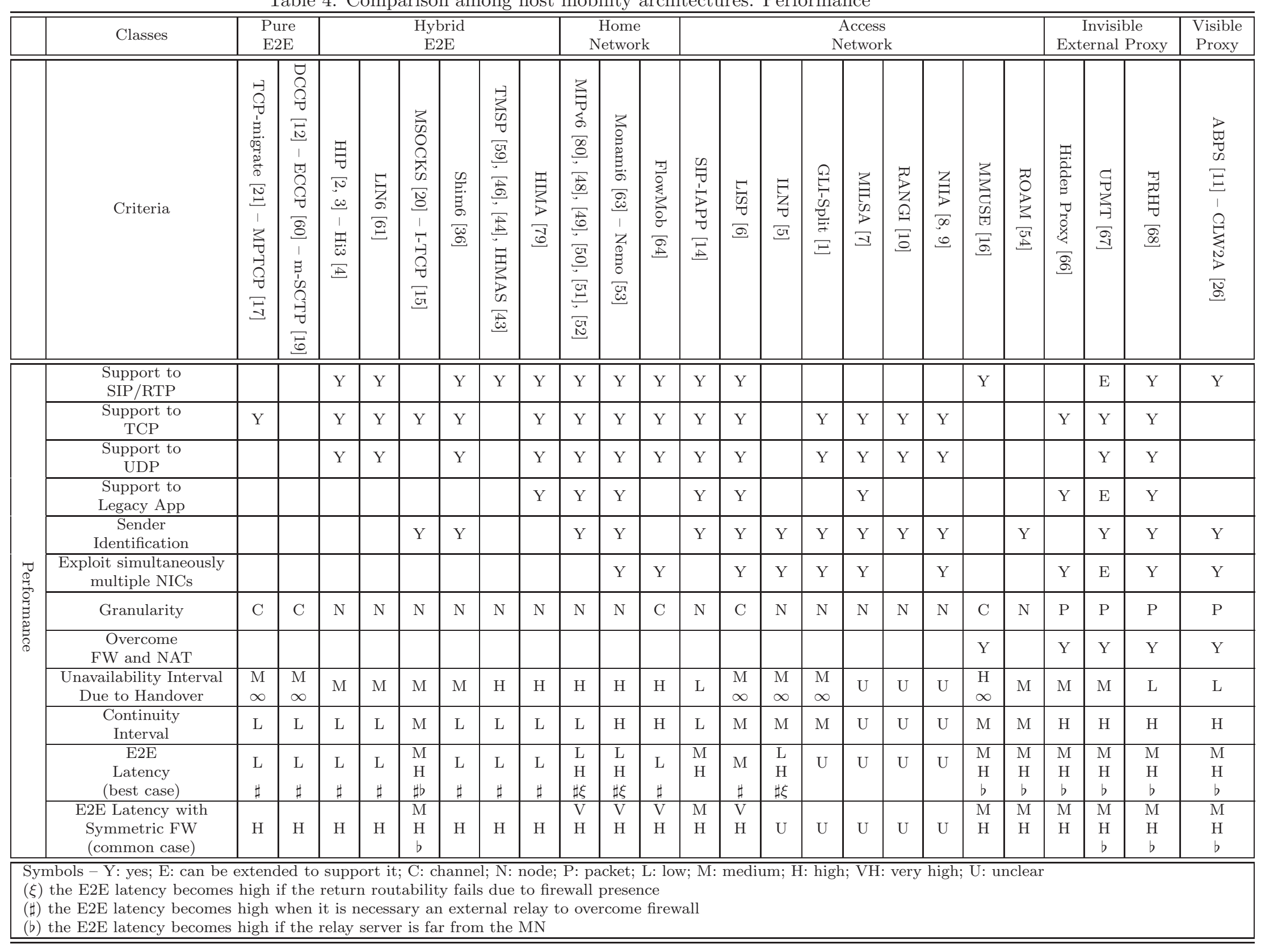




\subsection{General}

This subtable of Table 3 contains two criteria, i.e., "deployability" and "implementation".

\subsubsection{Deployability}

The deployability criterion ranks systems that can be effectively deployed on the current Internet. This feature is derived from the characteristics analyzed in the rest of the table.

In substance, system architectures are considered as deployable when they work over IPv4 networks, and do not require modifications to the backbone. In general, pure end-to-end solutions and those that resort to an external proxy are deployable. Some hybrid end-to-end approaches are deployed (MSOCKS, I-TCP, TMSP, HIMA), as well as some proposals that modify the access network only (however, one might argue that this requirement prevents the deployability of the systems in the real world), i.e. SIP-IAPP, [14], MMUSE, ROAM.

\subsubsection{Implementation}

The implementation feature indicates whether or not some real prototype implementation is available. Implementations or descriptions of implementation can be found for the following systems: ABPS [11]; DCCP [81]; FLowMob [82]; Hi3 [83]; Hidden Proxy [66]; HIP [84, 85]; I-TCP [15]; LIN6 [61, 75]; LISP [86, 87]; MCoA (monami6) and NEMO [88, 89, 90]; MIPv6 [80, 88, 89, 90]; MMUSE [91]; MPTCP [17, 18]; MSOCKS [20]; NIIA [92]; Shim6 [93, 85]; SIP-IAPP [13]; TCPmigrate [94]; TMSP [59]; UPMT [67, 95].

Below, we provide a brief description of the implementation software projects, for the mentioned architectures, available at the time of writing. The systems described are ordered alphabetically.

\section{$A B P S$}

A detailed description of the implementation of the ABPS system is reported in [11]. The software is available upon direct request to the authors. Current versions of ABPS are available for Linux and Android systems.

\section{$D C C P$}

DCCP-TP is an implementation of DCCP optimized for portability [81]. The site provides source code downloads and documentation. Such implementation includes many DCCP features, including IPv4 NAT encapsulation. It has been implemented in C language, for Linux systems, and released under the GNU Lesser General Public License (GPL) v2.1. 


\section{FLowMob}

In [82], an implementation for Linux operating systems of PMIP and its FlowMob extension was built to validate the proposal.

\section{Hi3}

In [83], a prototype implementation of the Hi3 software architecture is described. It has been conceived as a layered agent based architecture to separate the different functionalities of the system. The main result of such a development process was the validation of the $\mathrm{Hi} 3$ model architecture.

\section{Hidden Proxy}

A prototype implementation for Linux systems of the Hidden Proxy is described in [66]. The aim of the implementation was to assess the viability of the proposal. The software is available upon direct request to the authors.

\section{$H I P$}

The OpenHIP project is developing free, open source software implementing the HIP protocol [85]. The aim is to develop client software for Linux, BSD, Mac OS X, and Windows operating systems, plus some tools for experimenting with HIP (e.g., network analyzers).

Another IPv6 based implementation of HIP for Linux systems is presented in [84].

Finally, Hip4Inter.net was another effort for implementing the base HIP (whose Web site was unreachable at the moment of writing). FreeBSD and Linux were supported.

\section{$I-T C P$}

The paper presenting the I-TCP also provides a description of a prototype implementation [15]. This implementation includes modifications to the TCP and Mobile-IP code on the routers and MNs. Authors state that no modifications are needed in the Unix kernel in the MN. In the MN, the I-TCP library provides the API for the I-TCP functions which are similar to the socket related system calls in Unix. A further description of the implementation and a performance evaluation are reported in [96].

\section{LIN6}

In $[61,75]$, a prototype implementation of LIN6 on NetBSD/i386 is presented. The main goal of this effort was to validate the described system architecture. 


\section{LISP}

OpenLISP is an open source implementation of the LISP Protocol running in the kernel of the FreeBSD Operating System [86]. It implements a new type of sockets, called Mapping Sockets, providing an API that can be used by any user space process.

LISPmob is another open-source LISP implementation for Linux, Android and OpenWrt [87].

\section{MIPv6, MCoA monami6 and NEMO}

There are several available implementations of MIPv6 [80, 88, 89, 90]. However, only some examples taken from seminal articles will be presented here, together with their related projects.

UMIP is an open source implementation of Mobile IPv6 and NEMO Basic Support for Linux. It is released under the GPL v2 [89].

The Nautilus6 working group provided Linux and BSD reference implementations of IPv6 related libraries and IPv6 applications [88]. In particular, this working group developed SHISA (an implementation of Mobile IPv6 and NEMO), NEPL (a NEMO platform for Linux), and ATLANTIS (a NEMO Basic Support implementation for NetBSD).

The TAHI project was concluded at the end of 2012 by providing implementations of several protocols and test tools, e.g., IPv6 core, IPsec, DHCPv6, MIPv6, NEMO [90].

\section{MMUSE}

An implementation of MMUSE is available in [91]. The prototype is written in Java language (which makes it a multi-platform solution) and uses mjsip as SIP stack. The source code is released under the GPL v2.

\section{MPTCP}

Implementations of MPTCP for Linux systems are described in [17, 18]. The source code is available in [17]. Three modes of operation are available, i.e. i) the regular MPTCP, where all active NICs (and thus multiple TCP flows among two nodes) are used concurrently, ii) a backup mode, that uses only a subset of possible communication flows, iii) a single-path mode where a single sub flow is used; when the NIC goes down, a hard handover (break-before-make) is performed and a new TCP sub flow is created.

\section{$M S O C K S$}

The implementation of a MSOCKS library is described in [20]. It is a shim library that sits between the application and the kernel on the mobile node. Its task is to provide an interface to the application, which is identical to that of the 
Berkeley Sockets API, while internally using the normal TCP stack of the kernel to provide mobility functions. On Windows platforms, the MSOCKS library was designed to be a DLL that fits between the application and the WinSock DLL. Moreover, the paper mentions a BSD OS implementation, using the shared library support, but no further details are given.

NIIA

In [92], the authors describe a prototype implementation of NIIA, built based on the hip4inter.net code base.

\section{Shim6}

The OpenHIP project mentioned above also provides an implementation of Shim6 [85]. The idea was to provide an open-source Linux implementation of Shim6 that borrows from (and can later integrate with) the HIP implementation. This would lead to an eventual evolution towards a combined Shim6/HIP implementation.

LinShim6 (and its variant, MipShim6) is another project that implements Shim6 [93].

\section{TCP-migrate}

A Linux-based implementation of TCP-migrate has been released as free software, under the GPL v2 [94].

\section{TMSP}

In [59], an implementation on Linux kernel of TMSP is described, followed by a performance evaluation. The system was tested with video streaming and video conferencing applications.

\section{UPMT}

UPMT has been implemented for mainstream Linux and then ported on the Android OS [67, 97]. The source code is available under the GPL. For demonstration purposes, a UPMT Linux Live distribution is provided where both UPMT mobile and anchor nodes can be set up.

\subsection{Requirements}

The requirements subtable describes what protocols and features are necessary for each given architecture. In particular, we mark systems that require the presence of IPv6, MIPv6 or IPSec. As shown in Table 3, all solutions that exploit a software entity in the home network do require an IPv6 approach. Indeed, these solutions are designed to work with IPv6 or the like, and do exploit a HA.

Some systems in the hybrid end-to-end class, i.e. LIN6 and Shim6 (as their names suggest), employ IPv6 as well. The other approaches, that have been 
devised to work over IPv6 nets, require a separation between the identifier and locator, i.e. GLI-Split, ILNP, LISP, MILSA, NIIA, RANGI.

MIPv6 is needed by systems belonging to the Home Network class. These systems make use of IPsec, too. IPsec is used by MILSA and Hidden Proxy.

\subsection{Needs Modifications}

This subtable in Table 3 summarizes the changes that need to be introduced in the network entities of the examined architectures (e.g. infrastructures, protocols, terminals, applications) so that they can be deployed in real scenarios. These criteria are particularly important since they allow us to measure the applicability of a solution. In this way, the requirement for modifying a given entity prevents the simple deployment of the solution and its practical use.

The need for modifying the protocol stack at a MN (see the row "Protocol Stack in MN") is not a major limitation because the MN exploits the advantage of mobility, which is a valid reason for users to install some additional software in their MN. Indeed, almost all the considered systems do require some particular changes to the $\mathrm{MN}$, and if they do not, they still require the applications running on the MN to be modified (i.e. TMSP [59], HIMA [79], see the row "Applications in the MN"), or the access network to provide specific features and some border gateway to be present (i.e. SIP-IAPP, [14], see rows "Access Network" and "Border Gateways").

Conversely, modifying the $\mathrm{CN}$ to ease the mobility of other nodes (i.e. the MN) is not a convenient practice and it is very likely that those changes will never be deployed in real contexts. The list of systems that require such changes is reported in the row labeled "Protocol Stack in CN". Basically, systems that do not have this requirement, assume the presence of an additional relay/proxy or a border gateway that manage packets to be sent to the $\mathrm{CN}$.

Analogous considerations refer to those changes which are needed in the applications executed in the MN and in the CN (see rows "Applications in the MN" and "Applications in the CN"). Legacy applications cannot be modified. Owing to the above observations we can conclude that it would be difficult to see pure or hybrid end-to-end host mobility architectures being deployed in real, current scenarios. Moreover, we mentioned that systems in the access network class, that employ the identifier- locator distinction, are not designed for IPv4 networks.

Modifications to the network infrastructures require labour-intensive work, as well as reconfiguration and modification of the networks. This is particularly true when a solution needs to modify all the access networks or border gateways. As mentioned, rows "Access Network" and "Border Gateways" report those systems that have such requirements. They basically fall in the home network-based and access network-based host mobility classes. Thus, the deployment of home net- 
work-based and access network-based host mobility architectures suffers from a massive inertia, due to the difficulties of deploying them in the existing Internet.

Solutions that rely on an external relay/proxy server are deployable because they do not impact on the existing infrastructures (see the "Require External Relay" row in the table).

The above considerations represent an important claim. We are indeed arguing that it is quite unlikely that the great majority of well known systems will be employed in real scenarios, at least over a short or medium time period.

\subsection{Performance}

The Performance subtable in Table 4 consists of criteria that describe the QoS provided by the considered architectures. In particular, these criteria include: the ability of providing support to different applications and the protocols commonly employed to build them (i.e. SIP/RTP, UDP, TCP or legacy applications), characteristics in terms of service continuity, service unavailability, end-to-end latency, security, ability to exploit simultaneously all the available NICs and to switch granularity. When we refer to the support provided to some communication protocols (e.g. SIP, RTP, TCP), we mean that the considered system copes with the typical problems arising at that protocol level during a handover. It is thus evident that a particular scheme may provide support only to protocols working at a higher level of the network stack.

Of course, only multihoming systems (described in Section 4) may allow exploiting multiple NICs simultaneously ("Exploit simultaneously multiple NICs" row).

The row "granularity" reports whether or not a system works by considering i) the $\mathrm{MN}$ as a whole $(\mathrm{N})$, ii) a channel where a communication flow occurs between the MN and its CNs $(\mathrm{C})$, or iii) even when the communication management occurs on a per-packet basis (P). Again, systems in the same class work in a similar way. Thus, pure end-to-end systems work at the level of communication channels. Hybrid end-to-end systems and those that employ software on the home network operate at the level of the node (with the exception of FlowMob that work with channels). Systems employing specific features at the access network work at node or channel levels. Finally, solutions that employ some (visible or invisible) relay work on a per-packet basis.

It is worth noting that few systems are able to overcome the problems which may arise when NATs and firewalls are in the network. This is a typical feature of proxy-based approaches. However, the solutions that separate locators and identifiers, do not take into consideration the presence of NAT, since it is assumed that IPv6 is employed, thus NAT would not be used anymore. Moreover, the use of identifiers enables firewalls to have access control rules that are based on identity, rather than address or location. However, this requires further work and costs to 
the firewall; in the case of symmetric firewalls, every time an end-node creates a connection using a new identifier, the firewall must be instructed properly.

The unavailability interval measures the length of the interval time during which the MN is not able to communicate with the $\mathrm{CN}$, due to the handover. This metric is very important when some interactive application (e.g. VoIP) is executed. This unavailability interval includes the time needed to re-configure all the network entities involved in the end-to-end $\mathrm{MN}-\mathrm{CN}$ communication. For this reason, all the solutions based on MIPv6 suffer from a high unavailability interval (around 1-3 seconds, typically) because they require an intense packet exchange between the $\mathrm{MN}$, the home network and the $\mathrm{CN}$ to register the new CoA on the HA and to perform the return routability operation. However, if during the re-configuration of the preferred NIC, the MN exploits a different NIC, the unavailability interval decreases. For this reason, all the solutions employing multihoming on a per-packet basis limit the unavailability interval length.

In addition, it is very important to note that, commonly, the unavailability time interval does not include the handover time only. When a network becomes unavailable (and thus a handover is required) there is a time interval during which the NIC appears to be functioning (at the MN), but packets are lost [98]. Then, the MN only detects that the connection with its access points has been lost after a while and eventually the handover begins. If the MN is able to detect these losses and can retransmit packets immediately, by exploiting a different, already configured and properly working NIC (as it may happen when a multihoming solution is employed), then the unavailability interval decreases. For that reason, the solutions that adopt some "early packet loss detector", such as ABPS and FRHP, significantly reduce the unavailability interval. For the sake of completeness, in the row labeled "Unavailability Interval Due to Handover" in Table 4, the symbol $\infty$ means that the architecture may fail to complete the handover: as concerns pure end-to-end solutions, this happens when both MN and CN change their IP addresses at the same time; in access network-based solutions, this happens when the MN enters an access network whose border gateway is not able to support the host mobility.

The "continuity interval" row in Table 4 ranks the time during which the MN may communicate with the CN. In particular, it measures the distance between two consecutive unavailability intervals, in intermittent communication scenarios. This metric rewards those systems capable of switching seamlessly from the NIC in use to another NIC already working. Conversely, all the host mobility solutions that do not allow the simultaneous use of multiple NICs, would incur severe service interruptions; this corresponds to a low continuity of service level. Similarly, those architectures where handovers may fail (see the discussion of the unavailability interval above) may provide a low level of service continuity. Finally, the maximum 
continuity interval is provided by solutions based on the early packet loss detection; this is due to the fact that these approaches may recover from packet losses by switching to (and retransmitting through) a different NIC [98].

The "end-to-end latency" row measures the time needed to deliver a packet from the MN application to the $\mathrm{CN}$ application. This metric is very important for interactive applications. The lowest bound of this latency corresponds to a direct communication between the $\mathrm{MN}$ and its $\mathrm{CN}$, as in the case of pure and hybrid end-to-end host mobility solutions. The access network-based solutions do not introduce additional delays because the packets transit through an entity (the border gateway) that is already in the path between MN and CN. In home network-based solutions, on the other hand, the situation is more complex. Indeed, if the return routability operation is successful, then the communication between $\mathrm{MN}$ and $\mathrm{CN}$ is direct, with a consequent minimum latency. However, if this operation fails, then packets transit through the home network, hence increasing the average latency, depending on the distance between the $\mathrm{CN}$ and the home network, and between the home network and the MN. Solutions based on an external relay/proxy obviously add a delay, depending on the distance of the relay/proxy server. However, it is worth pointing out that in the real scenario there are often firewalls and NAT systems that block direct communication and impose the interposition of a relay. Thus, in this common situation there is a latency increase, except for the external relay-based solutions that already utilize a relay.

The last two rows in Table 4 report the end-to-end latency in the best scenario (with no firewalls) and in the most common scenario (with symmetric firewalls), respectively. In the most common scenario, all the solutions are almost equivalent in terms of end-to-end latency.

\section{Conclusions}

This work presents an overview of the main architectural solutions for managing the handover process in mobile wireless communications. All the examined schemes have been grouped and categorized according to their main characteristics, the features they offer and the level of network stack in which they operate.

This survey highlights that a number of issues need to be addressed when designing an architecture for mobile communications, even though the same issues may not be as obvious as it first appears. Our discussion focuses on the key issue of handover management, and concludes that, firstly, the best solution to this issue has not yet been identified. Indeed, all the schemes investigated in the literature present some advantages and drawbacks.

Secondly, at the time of writing, the most widespread approach for building software architectures that support users mobility is based on the use of MIPv6. 
The solutions that adopt this approach are not deployable in current IPv4 networks. Moreover, these solutions cannot coexist with symmetric firewalls.

Thirdly, we have shown that many techniques are not able to cope with some problems due to the presence of symmetric NATs (this is a main issue for strategies that might be deployed on the current IPv4 Internet) and symmetric firewalls placed in front of two end-systems, which prevents the two end-systems to communicate. Drawing on these considerations, we can say that the approaches that solve the problem through the use of external relays seem to be more promising.

Fourth, there are many popular applications and protocols that violate the protocol stack stratification, since they add information regarding the network and transport layers within application data payloads. A prominent example is SIP. These applications and protocols introduce several complications in the design of an effective mobility architecture. Indeed, to make the underlying system application/protocol-transparent, it is necessary to update the application payloads included in the transmitted packets. This is done to ensure that the IP address and port contained at the transport and network layers match those included within the application data. This task can be more easily accomplished by exploiting two proxies, a local one and an external one, working on top of the transport layer, rather than some network-layer solutions.

On the other hand, those applications and protocols that do not violate the protocol stratification can be easily supported by MIPv6 solutions. Note that this is true when only one network interface is used by the MN. As regards multihoming, the approaches may be subjected to poor performances in terms of introduced latencies and connection continuity intervals.

In conclusion, the above considerations suggest that it is quite unlikely that many of these proposals will be adopted in real life scenarios, at least on a shortor medium- term basis. Our claim is that future proposals should deal with issues concerned with the presence of NATs and firewalls natively. A good solution is to resort to dedicated services such as external proxies, that can be easily distributed on the Internet. As a matter of fact, many applications and their related protocols include the use of external proxies, although they are usually exploited for application purposes only. Some examples include VoIP applications, SIP-based applications and, in certain cases, HTTP-based applications [26]. We suggest that their use may be extended, in order to cater for issues concerned with mobility, as has already been proposed in the literature $[11,66]$.

As a concluding remark, it should be mentioned that cloud computing environments can be used as the infrastructure to dynamically set up (and release) the proxies on the server-side, in accordance with the pay-as-you-go principle of cloud based services [99]. 


\section{References}

[1] M. Menth, M. Hartmann, D. Klein, Global locator, local locator, and identifier split (gli-split), Future Internet 5 (1) (2013) 67-94.

[2] L. Bokor, L. T. Zeke, S. Nováczki, G. Jeney, Protocol design and analysis of a hip-based per-application mobility management platform., in: W. Li, A. Y. Zomaya, A. Al-Jumaily (Eds.), Proceedings of the Seventh ACM International Workshop on Mobility Management \& Wireless Access, MOBIWAC 2009, ACM, 2009, pp. 7-16.

[3] R. Moskowitz, P. Nikander, Host identity protocol (hip) architecture, RFC 4423 (Informational) (may 2006).

URL http://www. ietf.org/rfc/rfc4423.txt

[4] A. Gurtov, D. Korzun, A. Lukyanenko, P. Nikander, Hi3: An efficient and secure networking architecture for mobile hosts., Computer Communications 31 (10) (2008) 2457-2467.

[5] R. Atkinson, S. Bhatti, Identifier-Locator Network Protocol (ILNP) Architectural Description, RFC 6740 (Proposed Standard) (November 2012).

[6] D. Saucez, L. Iannone, O. Bonaventure, D. Farinacci, Designing a deployable internet: The locator/identifier separation protocol, IEEE Internet Computing 16 (6) (2012) 14-21. doi:http://doi.ieeecomputersociety.org/10.1109/MIC.2012.98.

[7] J. Pan, R. Jain, S. Paul, C. So-in, Milsa: A new evolutionary architecture for scalability, mobility, and multihoming in the future internet, IEEE Journal on Selected Areas in Communications 28 (8) (2010) 1344-1362. doi:10.1109/JSAC.2010.101012.

URL http://dx.doi.org/10.1109/JSAC. 2010.101012

[8] B. Ahlgren, J. Arkko, L. Eggert, J. Rajahalme, A node identity internetworking architecture, in: INFOCOM 2006. 25th IEEE International Conference on Computer Communications. Proceedings, 2006, pp. 1-6. doi:10.1109/INFOCOM.2006.51.

[9] S. Schütz, H. Abrahamsson, B. Ahlgren, M. Brunner, Design and implementation of the node identity internetworking architecture, Comput. Netw. 54 (7) (2010) 1142-1154. doi:10.1016/j.comnet.2009.10.015.

URL http://dx.doi.org/10.1016/j.comnet.2009.10.015 
[10] X. Xu, R. Jain, Routing architecture for the next generation Internet (RANGI), Internet-Draft draft-xu-rangi, IETF Secretariat (2009).

[11] V. Ghini, S. Ferretti, F. Panzieri, The "always best packet switching" architecture for sip-based mobile multimedia services, Journal of Systems and Software 84 (11) (2011) 1827 - 1851. doi:10.1016/j.jss.2011.06.025. URL http://www.sciencedirect.com/science/article/pii/S0164121211001506

[12] E. Kohler, M. Handley, S. Floyd, Datagram Congestion Control Protocol (DCCP), RFC 4340 (Proposed Standard), updated by RFCs 5595, 5596 (March 2006).

URL http://www.ietf.org/rfc/rfc4340.txt

[13] IEEE Recommended Practice for Multi-Vendor Access Point Interoperability via an Inter-Access Point Protocol Across Distribution Systems Supporting 802.11 Operation (Jan. 2002).

[14] C.-S. Wu, M.-T. Yang, K.-S. Hwang, Fast-handoff schemes for inter-subnet handoff in IEEE 802.11 WLANs for SIP/RTP applications, in: M. Guizani, H.-H. Chen, X. Zhang (Eds.), Proceedings of the International Conference on Wireless Communications and Mobile Computing, IWCMC 2007, ACM, 2007, pp. 146-151.

[15] A. Bakre, B. R. Badrinath, I-tcp: indirect tcp for mobile hosts, in: Distributed Computing Systems, 1995., Proceedings of the 15th International Conference on, 1995, pp. 136-143. doi:10.1109/ICDCS.1995.500012.

[16] S. Salsano, A. Polidoro, C. Mingardi, S. Niccolini, L. Veltri, Sip-based mobility management in next generation networks., IEEE Wireless Communications 15 (2) (2008) 92-99.

[17] Multipath tcp - linux kernel implementation, http://multipath-tcp.org/.

[18] C. Paasch, G. Detal, F. Duchene, C. Raiciu, O. Bonaventure, Exploring mobile/wifi handover with multipath tcp, in: Proceedings of the 2012 ACM SIGCOMM Workshop on Cellular Networks: Operations, Challenges, and Future Design, CellNet '12, ACM, New York, NY, USA, 2012, pp. 31-36. doi:10.1145/2342468.2342476.

URL http://doi.acm.org/10.1145/2342468.2342476

[19] L. Budzisz, J. Garcia, A. Brunstrom, R. Ferrús, A taxonomy and survey of sctp research, ACM Computing Surveys 44 (4) (2012) 18:1-18:36. doi:10.1145/2333112.2333113.

URL http://doi .acm.org/10.1145/2333112.2333113 
[20] D. Maltz, P. Bhagwat, Msocks: an architecture for transport layer mobility, in: INFOCOM '98. Seventeenth Annual Joint Conference of the IEEE Computer and Communications Societies. Proceedings. IEEE, Vol. 3, 1998, pp. 10371045 vol.3. doi:10.1109/INFCOM.1998.662913.

[21] A. Snoeren, H. Balakrishnan, F. Kaashoek, Reconsidering Internet Mobility, in: 8th Workshop on Hot Topics in Operating Systems, Elmau/Oberbayern, Germany, 2001.

[22] A. Gladisch, R. Daher, D. Tavangarian, Survey on mobility and multihoming in future internet, Wireless Personal Communications 74 (1) (2014) 45-81. doi:10.1007/s11277-012-0898-6.

URL http://dx.doi.org/10.1007/s11277-012-0898-6

[23] B. Ahlgren, C. Dannewitz, C. Imbrenda, D. Kutscher, B. Ohlman, A survey of information-centric networking, Communications Magazine, IEEE 50 (7) (2012) 26-36. doi:10.1109/MCOM.2012.6231276.

[24] H. Tuncer, Y. Nozaki, N. Shenoy, Virtual mobility domains - a mobility architecture for the future internet, in: Communications (ICC), 2012 IEEE International Conference on, 2012, pp. 2774-2779. doi:10.1109/ICC.2012.6363872.

[25] Y. Wang, J. Bi, C. Peng, H. Hu, Una: A new internet architecture for userlevel multi-homing and mobility, in: Proceedings of the 6th International Conference on Future Internet Technologies, CFI '11, ACM, New York, NY, USA, 2011, pp. 13-18. doi:10.1145/2002396.2002400.

URL http://doi.acm.org/10.1145/2002396.2002400

[26] S. Ferretti, V. Ghini, A web 2.0, location-based architecture for a seamless discovery of points of interests, in: The Fifth Advanced International Conference on Telecommunications, AICT 2009, 2009, pp. 226-231.

[27] G.-H. Tu, Y. Li, C. Peng, C.-Y. Li, H. Wang, S. Lu, Control-plane protocol interactions in cellular networks, SIGCOMM Comput. Commun. Rev. 44 (4) (2014) 223-234. doi:10.1145/2740070.2626302.

URL http://doi.acm.org/10.1145/2740070.2626302

[28] C.-T. Chou, K. G. Shin, An enhanced inter-access point protocol for uniform intra and intersubnet handoffs, IEEE Transactions on Mobile Computing 4 (4) (2005) 321-334. doi:http://doi.ieeecomputersociety.org/10.1109/TMC.2005.49. 
[29] V. Ghini, S. Ferretti, F. Panzieri, A strategy for best access point selection, in: Wireless Days (WD), 2010 IFIP, 2010, pp. 1-5. doi:10.1109/WD.2010.5657762.

[30] S. Pack, J. Choi, T. Kwon, Y. Choi, Fast-handoff support in ieee 802.11 wireless networks, Communications Surveys Tutorials, IEEE 9 (1) (2007) 212. doi:10.1109/COMST.2007.358968.

[31] I. Ramani, S. Savage, Syncscan: practical fast handoff for 802.11 infrastructure networks, in: INFOCOM 2005. 24th Annual Joint Conference of the IEEE Computer and Communications Societies. Proceedings IEEE, Vol. 1, 2005, pp. 675-684 vol. 1. doi:10.1109/INFCOM.2005.1497933.

[32] Ieee standard for information technology- local and metropolitan area networks- specific requirements- part 11: Wireless lan medium access control (mac) and physical layer (phy) specifications amendment 2: Fast basic service set (bss) transition, IEEE Std 802.11r-2008 (Amendment to IEEE Std 802.11-2007 as amended by IEEE Std 802.11k-2008) (2008) 1126doi:10.1109/IEEESTD.2008.4573292.

[33] R. Atkinson, S. Bhatti, S. Hailes, Evolving the internet architecture through naming, IEEE Journal on Selected Areas in Communications 28 (8) (2010) 1319-1325. doi:10.1109/JSAC.2010.101009.

URL http://dx.doi.org/10.1109/JSAC.2010.101009

[34] A. Gladisch, R. Daher, D. Tavangarian, Survey on mobility and multihoming in future internet, Wireless Personal Communications 74 (1) (2014) 45-81. doi:10.1007/s11277-012-0898-6.

URL http://dx.doi.org/10.1007/s11277-012-0898-6

[35] W. Ramirez, X. Masip-Bruin, M. Yannuzzi, R. Serral-Gracia, A. Martinez, M. S. Siddiqui, A survey and taxonomy of id/locator split architectures, Comput. Netw. 60 (2014) 13-33. doi:10.1016/j.bjp.2013.12.006.

URL http://dx.doi.org/10.1016/j.bjp.2013.12.006

[36] E. Nordmark, M. Bagnulo, Shim6: Level 3 Multihoming Shim Protocol for IPv6, RFC 5533 (Proposed Standard) (June 2009).

URL http://www.ietf.org/rfc/rfc5533.txt

[37] H. Tuncer, A. Kwasinski, N. Shenoy, Performance analysis of virtual mobility domain scheme vs. ipv6 mobility protocols, Computer Networks 57 (13) (2013) 2578-2596. doi:10.1016/j.comnet.2013.05.004.

URL http://dx.doi.org/10.1016/j.comnet.2013.05.004 
[38] J. Rosenberg, J. Weinberger, C. Huitema, R. Mahy, STUN - simple traversal of user datagram protocol (UDP) through network address translators (NATs), RFC 3489 (March 2003).

[39] J. Rosenberg, R. Mahy, C. Huitema, Traversal using relay NAT (TURN), Internet-Draft (September 2005).

[40] J. Rosenberg, Interactive Connectivity Establishment (ICE): A Protocol for Network Address Translator (NAT) Traversal for Offer/Answer Protocols, Tech. Rep. 5245, RFC Editor (Apr. 2010).

URL http://www.rfc-editor.org/rfc/rfc5245.txt

[41] D. Corujo, C. Guimaraes, B. Santos, R. L. Aguiar, Using an opensource ieee 802.21 implementation for network-based localized mobility management, Communications Magazine, IEEE 49 (9) (2011) 114-123. doi:10.1109/MCOM.2011.6011742.

[42] J. Rosenberg, H. Schulzrinne, G. Camarillo, A. Johnston, J. Peterson, R. Sparks, M. Handley, E. Schooler, SIP: Session initiation protocol (2002).

[43] P. Bellavista, A. Corradi, L. Foschini, Ims-compliant management of vertical handoffs for mobile multimedia session continuity, IEEE Communications Magazine 48 (2010) 114-121.

URL http://dl.acm.org/citation.cfm?id=1824609.1824623

[44] C. Kalmanek, J. Murray, C. Rice, B. Gessel, R. Kabre, A. Moskal, A networkbased architecture for seamless mobility services, Communications Magazine 44 (6) (2006) 103-109. doi:10.1109/MCOM.2006.1668427.

URL http://dx.doi.org/10.1109/MCOM.2006.1668427

[45] H. Schulzrinne, E. Wedlund, Application-layer mobility using sip, SIGMOBILE Mob. Comput. Commun. Rev. 4 (3) (2000) 47-57. doi:10.1145/372346.372369.

URL http://doi.acm.org/10.1145/372346.372369

[46] A. Udugama, K. Kuladinithi, C. Gorg, F. Pittmann, L. Tionardi, Netcape: Enabling seamless ims service delivery across heterogeneous mobile networks, Comm. Mag. 45 (7) (2007) 84-91. doi:10.1109/MCOM.2007.382665.

URL http://dx.doi.org/10.1109/MCOM. 2007.382665

[47] D. Johnson, C. Perkins, J. Arkko, RFC 3775:Mobility Support in IPv6 (Jun. 2004).

URL http://www.ietf.org/rfc/rfc3775.txt 
[48] N. Van Hanh, S. Ro, J. Ryu, Simplified fast handover in mobile ipv6 networks, Computer Communications 31 (15) (2008) 3594-3603. doi:10.1016/j.comcom.2008.06.009.

URL http://dx.doi.org/10.1016/j.comcom.2008.06.009

[49] H. Soliman, C. Castelluccia, K. Malki, L. Bellier, Hierarchical Mobile IPv6 mobility management (HMIPv6), RFC 4140, IETF (August 2005).

[50] S. Gundavelli, K. Leung, V. Devarapalli, K. Chowdhury, B. Patil, Proxy Mobile IPv6, RFC 5213 (Proposed Standard) (August 2008).

URL http://www.ietf.org/rfc/rfc5213.txt

[51] H. Zhou, H. Zhang, Y. Qin, H.-C. Wang, H.-C. Chao, A proxy mobile ipv6 based global mobility management architecture and protocol, Mobile Networks and Applications 15 (4) (2010) 530-542. doi:10.1007/s11036-009-01852 .

URL http://dx.doi.org/10.1007/s11036-009-0185-2

[52] W. He, I.-R. Chen, D.-C. Wang, Cross-layer integrated mobility and service management utilizing smart routers in mobile ipv6 systems, in: Proceedings of the 8th International Conference on Advances in Mobile Computing and Multimedia, MoMM '10, ACM, New York, NY, USA, 2010, pp. 140-147. doi:10.1145/1971519.1971545.

URL http://doi .acm.org/10.1145/1971519.1971545

[53] E. Perera, V. Sivaraman, A. Seneviratne, Survey on network mobility support, ACM SIGMOBILE Mobile Computing and Communications Review 8 (2) (2004) 7-19. doi:10.1145/997122.997127.

URL http://doi.acm.org/10.1145/997122.997127

[54] S. Zhuang, K. Lai, I. Stoica, R. Katz, S. Shenker, Host mobility using an internet indirection infrastructure, Wirel. Netw. 11 (6) (2005) 741-756. doi:10.1007/s11276-005-3528-3.

URL http://dx.doi.org/10.1007/s11276-005-3528-3

[55] K.-S. Kong, W. Lee, Y.-H. Han, M.-K. Shin, H. You, Mobility management for all-ip mobile networks: mobile ipv6 vs. proxy mobile ipv6, Wireless Communications, IEEE 15 (2) (2008) 36-45. doi:10.1109/MWC.2008.4492976.

[56] D. Klein, M. Hartmann, M. Menth, Nat traversal for lisp mobile node, in: Proceedings of the Re-Architecting the Internet Workshop, ReARCH '10, ACM, New York, NY, USA, 2010, pp. 8:1-8:6. doi:10.1145/1921233.1921243. URL http://doi .acm.org/10.1145/1921233.1921243 
[57] R. Whittle, S. Russert, TTR Mobility Extensions for Core-Edge Separation Solutions to the Internet's Routing Scale Problem, Tech. rep. (2008).

URL http://www.firstpr.com.au/ip/ivip/TTR-Mobility.pdf

[58] I. Stoica, D. Adkins, S. Zhuang, S. Shenker, S. Surana, Internet indirection infrastructure, IEEE/ACM Transactions on Networking 12 (2) (2004) 205218. doi:10.1109/TNET.2004.826279.

URL http://dx.doi.org/10.1109/TNET. 2004.826279

[59] T. M. Lim, C. K. Yeo, F. Lee, Q. V. Le, Tmsp: Terminal mobility support protocol, IEEE Transactions on Mobile Computing 8 (6) (2009) 849-863.

[60] M. Arye, E. Nordstrom, R. Kiefer, J. Rexford, M. Freedman, A formallyverified migration protocol for mobile, multi-homed hosts, in: Network Protocols (ICNP), 2012 20th IEEE International Conference on, 2012, pp. 1-12. doi:10.1109/ICNP.2012.6459961.

[61] F. Teraoka, M. Ishiyama, M. Kunishi, LIN6: A solution to mobility and multi-homing in IPv6, Internet-Draft draft-teraoka-ipng-lin6-02.txt, IETF Secretariat, Fremont, CA, USA (Jun. 2003).

URL http://www.rfc-editor .org/internet-drafts/draft-teraoka-ipng-lin6-02.txt

[62] J.-L. Lin, J.-Y. Pan, Hand-around: A handoff evolution with monami6, in: Wireless Communications, Networking and Mobile Computing, 2007. WiCom 2007. International Conference on, 2007, pp. 1775-1778. doi:10.1109/WICOM.2007.445.

[63] T. Narten, E. Nordmark, W. Simpson, H. Soliman, Neighbor Discovery for IP version 6 (IPv6), RFC 4861 (Draft Standard), updated by RFC 5942 (Sep. 2007).

URL http: //www.ietf.org/rfc/rfc4861.txt

[64] U. Toseef, A. Udugama, C. Goerg, C. Fan, F. Pittmann, Realization of multiple access interface management and flow mobility in ipv6, in: Proceedings of the 1st international conference on MOBILe Wireless MiddleWARE, Operating Systems, and Applications, MOBILWARE '08, ICST (Institute for Computer Sciences, Social-Informatics and Telecommunications Engineering), ICST, Brussels, Belgium, Belgium, 2007, pp. 26:1-26:8.

URL http://dl .acm.org/citation.cfm?id=1361492.1361524

[65] M. O'Dell, GSE: An Alternate Addressing Architecture for IPv6, InternetDraft draft-ipng-gseaddr-00.txt, IETF Secretariat (Feb. 1997). 
[66] V. Ghini, S. Cacciaguerra, F. Panzieri, P. Salomoni, A hidden proxy for seamless and abc multimedia mobile blogging, in: Proceedings of the 3rd IEEE Consumer Communications and Networking Conference (CCNC).

[67] M. Bonola, S. Salsano, A. Polidoro, Upmt: Universal per-application mobility management using tunnels, in: Proceedings of the 28th IEEE Conference on Global Telecommunications, GLOBECOM'09, IEEE Press, Piscataway, NJ, USA, 2009, pp. 2811-2818.

URL http://dl.acm.org/citation. cfm?id=1811681.1811847

[68] E. Giordano, L. Codecà, B. Geffon, G. Grassi, G. Pau, M. Gerla, Movit: The mobile network virtualized testbed, in: Proceedings of the Ninth ACM International Workshop on Vehicular Inter-networking, Systems, and Applications, VANET '12, ACM, New York, NY, USA, 2012, pp. 3-12. doi:10.1145/2307888.2307892.

URL http://doi .acm.org/10.1145/2307888.2307892

[69] R. Wakikawa, V. Devarapalli, G. Tsirtsis, T. Ernst, K. Nagami, I. Netcore, Multiple care-of-address registration, Tech. rep. (2003).

[70] J.-Y. Pan, J.-L. Lin, K.-F. Pan, Multiple care-of addresses registration and capacity-aware preference on multi-rate wireless links, Advanced Information Networking and Applications Workshops, International Conference on 0 (2008) 768-773. doi:http://doi.ieeecomputersociety.org/10.1109/WAINA.2008.154.

[71] B. Sousa, M. Silva, K. Pentikousis, M. Curado, A multiple care of addresses model, 2013 IEEE Symposium on Computers and Communications (ISCC) 0 (2011) 485-490. doi:http://doi.ieeecomputersociety.org/10.1109/ISCC.2011.5983884.

[72] X. Chen, H. Zhang, Y.-C. Chang, H.-C. Chao, Experimentation and performance analysis of multi-interfaced mobile router scheme, Simulation Modelling Practice and Theory 18 (4) (2010) 407 - 415. doi:http://dx.doi.org/10.1016/j.simpat.2009.09.005.

URL http://www. sciencedirect.com/science/article/pii/S1569190X09001294

[73] V. Zafeiris, E. A. Giakoumakis, Optimized traffic flow assignment in multihomed, multi-radio mobile hosts., Computer Networks 55 (5) (2011) 11141131.

[74] R. Atkinson, S. Bhatti, S. Hailes, A proposal for unifying mobility with multihoming, nat, \& security, in: Proceedings of the 5th ACM International Workshop on Mobility Management and Wireless Access, MobiWac '07, ACM, New 
York, NY, USA, 2007, pp. 74-83. doi:10.1145/1298091.1298105.

URL http://doi.acm.org/10.1145/1298091.1298105

[75] M. Kunishi, M. Ishiyama, K. Uehara, H. Esaki, F. Teraoka, Lin6: A new approach to mobility support in ipv6, Proceedings of the Third Inernational Sympsium on Wireless Personal Multimedia Communications (2000) 43.

[76] P. Nikander, J. Arkko, B. Ohlman, Host identity indirection infrastructure (Hi3), in: Proc. Second Swedish National Computer Networking Workshop (SNCNW), Karlstad, Sweden, 2004.

[77] J. Arkko, I. van Beijnum, Failure Detection and Locator Pair Exploration Protocol for IPv6 Multihoming, RFC 5534 (Proposed Standard) (June 2009). URL http://www. ietf.org/rfc/rfc5534.txt

[78] E. Kohler, Datagram Congestion Control Protocol Mobility and Multihoming, IETF, [Internet Draft] (Jul. 2004).

URL http://www .icir.org/kohler/dcp/draft-kohler-dccp-mobility-00.txt

[79] N. Shenoy, A framework for seamless roaming across heterogeneous next generation wireless networks, Wireless Networks 11 (6) (2005) 757-774. doi:10.1007/s11276-005-3529-2.

URL http://dx.doi.org/10.1007/s11276-005-3529-2

[80] Q. Li, T. Jinmei, K. Shima, Mobile IPv6: Protocols and Implementation, Morgan Kaufmann Publishers Inc., San Francisco, CA, USA, 2009.

[81] Dccp-tp home page, http://www.phelan-4.com/dccp-tp/tiki-index.php.

[82] T. Melia, C. J. Bernardos, A. de la Oliva, F. Giust, M. Calderón, Ip flow mobility in pmipv6 based networks: Solution design and experimental evaluation., Wireless Personal Communications (4) 603-627.

[83] G. Varela, A. Paz-Lpez, S. Vazquez-Rodriguez, R. Duro, Hi3 project: Design and implementation of the lower level layers, in: Virtual Environments, Human-Computer Interfaces and Measurement Systems, 2007. VECIMS 2007. IEEE Symposium on, 2007, pp. 36-41. doi:10.1109/VECIMS.2007.4373924.

[84] M. Komu, M. Kousa, J. Lundberg, C. Candolin, An implementation of hip for linux, in: Proceedings of Ottawa Linux Symposium, Ottawa, Canada, 2003.

[85] Openhip on sourceforge, http://sourceforge.net/projects/openhip/files/.

[86] The openlisp project [cited 2015].

URL http://www.openlisp.org/ 
[87] The lispmob project [cited 2015].

URL http://lispmob.org/

[88] Nautilus6 - network mobility website (2005) [cited 2014].

URL http://www.nautilus6.org

[89] Umip - mobile ipv6 and nemo for linux [cited 2014].

URL http://umip.org/

[90] Tahi project web site [cited 2014].

URL http://www.tahi.org/

[91] Mmuse: Mobility management using sip extensions web site [cited 2015].

URL http://netgroup.uniroma2.it/twiki/bin/view.cgi/Netgroup/MMUSEProject

[92] S. Schütz, H. Abrahamsson, B. Ahlgren, M. Brunner, Design and implementation of the node identity internetworking architecture, Computer Networks 54 (7) (2010) 1142 - 1154. doi:http://dx.doi.org/10.1016/j.comnet.2009.10.015.

URL http://www.sciencedirect.com/science/article/pii/S1389128609003363

[93] S. Barré, J. Ronan, O. Bonaventure, Implementation and evaluation of the shim6 protocol in the linux kernel, Comput. Commun. 34 (14) (2011) 16851695. doi:10.1016/j.comcom.2011.03.005.

URL http://dx.doi.org/10.1016/j.comcom.2011.03.005

[94] The migrate internet mobility project, http://nms.lcs.mit.edu/software/migrate/index.html.

[95] Upmt project homepage [cited 2014]. URL http://netgroup.uniroma2.it/twiki/bin/view.cgi/Netgroup/UpMT

[96] A. Bakre, B. Badrinath, Implementation and performance evaluation of indirect tcp, IEEE Transactions on Computers 46 (3) (1997) 260-278. doi:10.1109/12.580423.

[97] Upmt web site: http://netgroup.uniroma2.it/upmt.

[98] S. Ferretti, V. Ghini, M. Marzolla, F. Panzieri, Modeling the always best packet switching mechanism, in: Proceedings of the 6th International Conference on Next Generation Mobile Applications, Services and Technologies (NGMAST2012), IEEE, 2012.

[99] S. Ferretti, V. Ghini, F. Panzieri, E. Turrini, Seamless support of multimedia distributed applications through a cloud, in: Cloud Computing (CLOUD), 2010 IEEE 3rd International Conference on, 2010, pp. 548-549. doi:10.1109/CLOUD.2010.16. 